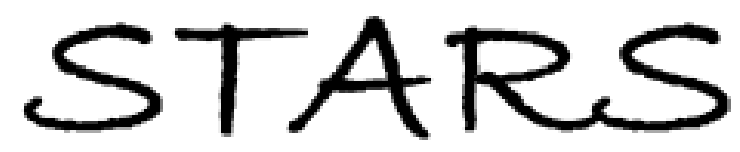

University of Central Florida

STARS

$1-1-2006$

\title{
On value distribution theory of second order periodic ODEs, special functions and orthogonal polynomials
}

Yik-Man Chiang

Mourad E. H. Ismail

University of Central Florida

Find similar works at: https://stars.library.ucf.edu/facultybib2000

University of Central Florida Libraries http://library.ucf.edu

This Article is brought to you for free and open access by the Faculty Bibliography at STARS. It has been accepted for inclusion in Faculty Bibliography 2000 s by an authorized administrator of STARS. For more information, please contact STARS@ucf.edu.

\section{Recommended Citation}

Chiang, Yik-Man and Ismail, Mourad E. H., "On value distribution theory of second order periodic ODEs, special functions and orthogonal polynomials" (2006). Faculty Bibliography 2000s. 4673.

https://stars.library.ucf.edu/facultybib2000/4673

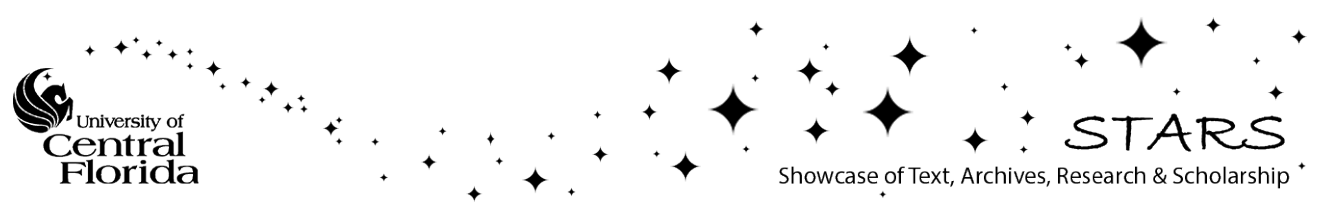




\title{
On Value Distribution Theory of Second Order Periodic ODEs, Special Functions and Orthogonal Polynomials
}

\author{
Dedicated to the memory of Noel Baker, a dear friend.
}

Yik-Man Chiang and Mourad E. H. Ismail

\begin{abstract}
We show that the value distribution (complex oscillation) of solutions of certain periodic second order ordinary differential equations studied by Bank, Laine and Langley is closely related to confluent hypergeometric functions, Bessel functions and Bessel polynomials. As a result, we give a complete characterization of the zero-distribution in the sense of Nevanlinna theory of the solutions for two classes of the ODEs. Our approach uses special functions and their asymptotics. New results concerning finiteness of the number of zeros (finite-zeros) problem of Bessel and Coulomb wave functions with respect to the parameters are also obtained as a consequence. We demonstrate that the problem for the remaining class of ODEs not covered by the above "special function approach" can be described by a classical Heine problem for differential equations that admit polynomial solutions.
\end{abstract}

\section{Introduction and Main Results}

We consider value distribution problems of the solutions of certain ordinary differential equations of the form

$$
f^{\prime \prime}(z)+A(z) f(z)=0
$$

where

$$
A(z)=B\left(e^{z}\right), \quad B(\zeta)=\frac{K_{k}}{\zeta^{k}}+\cdots+K_{0}+\cdots+K_{\ell} \zeta^{\ell}, \quad K_{\ell} K_{k} \neq 0 .
$$

Such equations always admit entire solutions. We recall that the order of $f$ is defined by $\sigma(f)=\lim \sup _{r \rightarrow+\infty} \log \log M(r, f) / \log r$, where $M(r, f)=\max _{|z|=r}|f(z)|$ denotes the maximum modulus of $f$. The "density" of the zeros of $f$ is given by $\lambda(f)=\lim \sup _{r \rightarrow+\infty} \log n(r, f) / \log r$, known as the exponent of convergence of $f$, where $n(r, f)$ denote the number of the zeros of $f$ in $|z|<r$. It is elementary to use Nevanlinna theory $[25,34]$ to deduce that any solution $f$ of $(1.1)$, where $A(z)$ can

Received by the editors February 4, 2004; revised June 2, 2005.

The research of the first author was partially supported by the University Grants Council of the Hong Kong Special Administrative Region, China (HKUST711/96P and HKUST6135/01P). The second author was partially supported by the NSF grant DMS 99-70865 and the Liu Bie Ju Center of Mathematical Sciences at the City University of Hong Kong.

AMS subject classification: 34M10, 33C15, 33C47.

Keywords: Complex Oscillation theory, Exponent of convergence of zeros, zero distribution of Bessel and Confluent hypergeometric functions, Lommel transform, Bessel polynomials, Heine Problem.

(c) Canadian Mathematical Society 2006. 
be any entire function of finite order, must have $\sigma(f)=+\infty$ and in [6,9] the conditions on $A(z)$ under which we have $\lambda(f)=+\infty$ were investigated. On the other hand, there exist exceptional cases of $A(z)$ where some solution $f$ of (1.2) satisfies

$$
\lambda(f)<\sigma(f)=+\infty .
$$

Bank and Laine obtained the following remarkable result [7]:

Theorem $\boldsymbol{A}$ Let $A(z)$ be an entire function given by (1.2). Suppose that the equation (1.1) admits a non-trivial solution $f$ that satisfies (1.3). Then there exist complex constants $d, d_{j}$, and a polynomial $\psi(\zeta)$ with only simple roots, such that if $\ell$ is an odd positive integer in (1.2), then $k=0$, and

$$
f(z)=\psi\left(e^{z / 2}\right) \exp \left(\sum_{j=0}^{\ell} d_{j} e^{j z / 2}+d z\right),
$$

where $d_{j}=0$ whenever $j$ is even. While if $\ell$ in (1.2) is an even positive integer, then $k$ is also even, and

$$
f(z)=\psi\left(e^{z}\right) \exp \left(\sum_{j=k / 2}^{\ell / 2} d_{j} e^{j z}+d z\right) .
$$

Remark 1.1 The statement of Theorem A in the present form is given in [14, Proposition 1.1] which makes precise the classification of the forms of (1.4), and (1.5) according to $f(z)$ and $f(z+2 \pi i)$ being linearly independent or linearly dependent, respectively.

It appears to be a difficult problem to determine how to choose $B(\zeta)$ in (1.2) so that the equation (1.1) would admit a solution $f$ with $\lambda(f)<+\infty$, and to write down such a solution explicitly $[4,5,8,13,14]$. The main difficulty is to determine the existence of the polynomial component $\psi(\zeta)$ that appears in the representations (1.4) or (1.5). In fact, Bank proposed a method of approximate square-root to solve this problem [4]. However, the complete solution to this problem is known only for some special cases of (1.2), such as the following theorem due to Bank, Laine and Langley [8]:

Theorem $B$ Let $K$ be a non-zero complex number. The equation

$$
f^{\prime \prime}+\left(e^{z}-K\right) f=0
$$

admits a non-trivial solution $f$ that satisfies (1.3) if and only if

$$
K=\frac{(2 n+1)^{2}}{16},
$$

for some non-negative integer $n$, and

$$
f(z)=\psi\left(e^{z / 2}\right) \exp \left(d e^{z / 2}-\frac{2 n+1}{4} z\right)
$$


where $d^{2}+4=0$ and

$$
\psi(\zeta)=\sum_{j=0}^{n} c_{j} \zeta^{j} .
$$

The first main purpose of this article is to show that the two well-known special cases of (1.1) are closely related to special functions. In fact, it is well known that the equation (1.6) can be transformed to a Bessel equation, e.g., [28]. Hence the general solution of (1.6) can be written in terms of Bessel functions. However, it is not clear how this can lead to the solution (1.8) as stated in Theorem B. We give a complete solution to this problem by showing directly how the general solution to (1.6) can be reduced to (1.8) when (1.3) holds. We prove (1.3) holds if and only if a very special relation of Bessel functions holds at the same time. In particular, we identify the polynomial $\psi(\zeta)$ that appears in (1.4) as the reverse Bessel polynomials $\theta_{n}(\zeta)$.

Our first generalization is

Theorem 1.2 Let $K_{0}$ and $K_{1}$ be complex constants. Then the equation

$$
f^{\prime \prime}+\left(K_{1} e^{z}-K_{0}\right) f=0
$$

admits linearly independent solutions

$$
y_{ \pm}(z)=A_{ \pm} J_{2 \sqrt{K_{0}}}\left( \pm 2 \sqrt{K_{1}} e^{z / 2}\right)+B_{ \pm} Y_{2 \sqrt{K_{0}}}\left( \pm 2 \sqrt{K_{1}} e^{z / 2}\right)
$$

Each of the solutions of (1.11) has a finite exponent of convergence of zeros if and only if

$$
2 \sqrt{K_{0}}=n+\frac{1}{2}
$$

where $n$ is an integer, and, if $n \geq 0$, then

$$
y_{ \pm}(z)=\theta_{n}\left(\mp 2 i \sqrt{K_{1}} e^{z / 2}\right) \exp \left( \pm 2 i \sqrt{K_{1}} e^{z / 2}+d z\right)
$$

where $\theta_{n}(x)$ is the reverse Bessel polynomial of degree $n$ and

$$
d=(-2 n-1) / 4 \text {. }
$$

If, however, $n=-m, m \geq 1$, then

$$
y_{ \pm}(z)=\theta_{m-1}\left(\mp 2 i \sqrt{K_{1}} e^{z / 2}\right) \exp \left( \pm 2 i \sqrt{K_{1}} e^{z / 2}+d^{\prime} z\right)
$$

where

$$
d^{\prime}=(-2 m+1) / 4
$$


Here the notation $J_{\nu}(z)$ and $Y_{\nu}(z)$ denote, respectively, the Bessel functions of the first and second kinds of order $\nu$ (see $[1,2,40])$. They are two linearly independent solutions of the Bessel equation

$$
x^{2} y^{\prime \prime}(x)+x y^{\prime \prime}(x)+\left(x^{2}-\nu^{2}\right) y(x)=0
$$

where both $\nu$ and $x$ can take complex values. Our standard reference for Bessel functions is Watson's monumental book [40] (see also [1, 2, 28, 42]). The reverse Bessel polynomials $\theta_{n}(z)$ are related to the Bessel polynomials $y_{n}(z)$ which are orthogonal with respect to the unit circle $|z|=1$. (See [33] and $\S 4, \S 10$ in this paper. See also [23].) The transformation (3.1) which transforms the equation (1.10) to (1.15) was actually first given by Lommel in 1871 (see [35], [40, p. 97] and $\S 3$ ).

Key to the proof of the Theorem 1.2 is the question of when will the expression

$$
A J_{\nu}(\zeta)+B Y_{\nu}(\zeta)
$$

admit only a finite number of zeros? Here $A$ and $B$ are coefficients to be determined. We call this the finite-zeros problem for (1.16). We shall give a complete characterization to this problem (Theorem 10.1). It appears that part of this result for Bessel functions is new. More detailed discussion will be given in $\S 10$.

In addition to Theorem A, Bank and Laine [7] also proved the following result:

Theorem $C$ Let $K$ be a non-zero complex number. Suppose that the equation

$$
f^{\prime \prime}+\left(-\frac{1}{4} e^{-2 z}+\frac{1}{2} e^{-z}+K\right) f=0
$$

admits a non-trivial solution $f$ that satisfies (1.3). Then there exists a positive integer $n$ with $K=-n^{2}$ and $f$ is equal to either

$$
f_{1}(z)=\left(\sum_{j=0}^{-(n-1)} B_{j} e^{j z}\right) \exp \left(\frac{1}{2} e^{-z}+n z\right)
$$

or

$$
f_{2}(z)=\left(\sum_{j=0}^{-n} b_{j} e^{j z}\right) \exp \left(-\frac{1}{2} e^{-z}+n z\right)
$$

Conversely, for each positive integer $n$, the equation (1.17) with $K=-n^{2}$ possesses two linearly independent solutions of the above forms.

We are able to use the Lommel transformation again to obtain a complete solution (see Theorems 1.3 and 1.6 below) to another sub-class of (1.1) with $A(z)$ given by (1.2) which contains (1.17) as a special case. It appears that many authors were not aware that the above equation (1.17) was already noted by Kamke in [31, pp. 36, 404] 
(see also [11, p. 36]) and that it can be transformed, via the Lommel transformation again $(\S 3, \S 7)$, to the Coulomb wave equation

$$
\frac{d^{2} y}{d x^{2}}+\left(1-\frac{2 \eta}{x}-\frac{L(L+1)}{x^{2}}\right) y=0,
$$

where $L, \eta$ are complex constants. The Coulomb wave equation is a form of confluent hypergeometric equation (see $\S 10$ and $[1,2])$. We denote two linearly independent solutions of $(1.20)$ by $F_{L}(\eta, x), G_{L}(\eta, x)$ to be the regular and irregular Coulomb wave functions, respectively (see $\S 6$ ). We extend Theorem C to:

Theorem 1.3 Let $K$ be a complex constant. Then

$$
f_{ \pm}(z)=A_{ \pm} F_{L}\left( \pm \frac{i}{2}, \pm \frac{i}{2} e^{-z}\right)+B_{ \pm} G_{L}\left( \pm \frac{i}{2}, \pm \frac{i}{2} e^{-z}\right),
$$

are linearly independent solutions of the equation (1.17), where

$$
L=\frac{1}{2}(-1+2 i \sqrt{K}) .
$$

Moreover, each solution of (1.21) has a finite exponent of convergence of zeros if and only if

$$
L=n+\frac{1}{2} \quad \text { or equivalently } \quad K=-\left(L+\frac{1}{2}\right)^{2}=-n^{2}
$$

for some positive integer $n$, and

$$
f_{+}(z)=y_{n}\left(e^{z} ; 1,1\right) \exp \left(-\frac{1}{2} e^{-z}\right)
$$

and

$$
f_{-}(z)=e^{z} y_{n-1}\left(e^{z} ; 3,-1\right) \exp \left(\frac{1}{2} e^{-z}\right) .
$$

Remark 1.4 The polynomials $y_{n}(x ; a, b)$, where $a, b$ are constants, in the above theorem are known as the generalized Bessel polynomials of degree $n$ in $x$ with parameters $a$ and $b$. The Bessel polynomials (and hence the reverse Bessel polynomials) mentioned in $\S 1$ in connection with Theorem 1.2 are special cases of the generalized Bessel polynomials which are orthogonal on the unit circle. They will be defined in $\S 4$.

Remark 1.5 According to Remark 4.2 below, the above parameter $a$ of the generalized Bessel polynomial in Remark 1.4 is not equal to a negative integer in (1.23) and (1.24), so that the polynomials $y_{n}$ and $y_{n-1}$ have degrees exactly $n$ and $n-1$, respectively. 
In fact, Theorem 1.3 (and hence Theorem C) is a simple corollary of the following theorem.

Theorem 1.6 Let $K_{-2}, K_{-1}$ and $K_{0}$ be complex numbers such that $K_{-2} K_{-1} \neq 0$. Then the differential equation

$$
f^{\prime \prime}+\left(K_{-2} e^{-2 z}+K_{-1} e^{-z}+K_{0}\right) f=0,
$$

admits linearly independent solutions

$$
f_{ \pm}(z)=A_{ \pm} F_{L}\left(\eta_{ \pm}, \alpha_{ \pm} e^{-z}\right)+B_{ \pm} G_{L}\left(\eta_{ \pm}, \alpha_{ \pm} e^{-z}\right),
$$

where

$$
\alpha_{ \pm}^{2}=K_{-2}, \quad-2 \eta_{ \pm} \alpha_{ \pm}=K_{-1}, \quad L=\frac{1}{2}\left(-1+2 i \sqrt{K_{0}}\right)
$$

Each of the solutions of (1.26) satisfies (1.3) if and only if there are non-negative integers $n_{+}$and $n_{-}$such that $n_{+}>n_{-} \geq 0$ and

$$
i\left(\sqrt{K_{0}} \pm \frac{K_{-1}}{2 \sqrt{K_{-2}}}\right)=n_{ \pm}+\frac{1}{2}
$$

or equivalently

$$
\frac{i K_{-1}}{\sqrt{K_{-2}}}=n_{+}-n_{-} \quad \text { and } \quad 2 i \sqrt{K_{0}}=n_{+}+n_{-}+1
$$

By writing $n=n_{+}, \hat{n}=n_{+}-n_{-}$, we have

$$
f_{+}(z)=e^{z / 2} e^{-\left(1-a_{+} / 2\right) z} y_{n}\left(e^{z} ; a_{+}, b_{+}\right) \exp \left(-\frac{b_{+}}{2} e^{-z}\right)
$$

and

$$
f_{-}(z)=e^{z / 2} e^{-\left(1-a_{-} / 2\right) z} y_{(n-\hat{n})}\left(e^{z} ; a_{-}, b_{-}\right) \exp \left(-\frac{b_{-}}{2} e^{-z}\right)
$$

where $a_{ \pm}=2\left(1+i \eta_{ \pm}\right)$and $b_{ \pm}=-2 i \alpha_{ \pm}$.

Remark 1.7 We note that since $K_{-1} \neq 0$, it follows from (1.29) that the integer $\hat{n}=n_{+}-n_{-}$cannot be zero. We can deduce from this observation that the two solutions (1.30) and (1.31) cannot both be zero-free, as opposed to the case of the solutions (1.13) of the equation (1.10) where both linearly independent solutions there are zero-free when $n=0$. We also note that none of the subscripts $n$ and $n-\hat{n}$ in (1.30) and (1.31) respectively are allowed to be negative.

Remark 1.8 The generalized Bessel polynomial $y_{n}(\zeta ; a, b)$ will have a degree less than $n$ if $a$ equals a negative integer as will be explained in Remark 4.2 below. 
The main idea of the proof of Theorem 1.6 is similar to that of Theorem 1.2. We want to characterize when the expression

$$
A F_{L}(\eta, \zeta)+B G_{L}(\eta, \zeta)
$$

will admit only a finite number of zeros in the complex plane. Here $A$ and $B$ are coefficients to be determined. We call this the finite-zeros problem of (1.32). We show that this can happen if and only if a very special relation holds amongst the parameters $L$ and $\eta$ (Theorem 10.2). In this case the corresponding polynomial component that appears in (1.5) where (1.3) is satisfied turns out to be a generalized Bessel polynomial. Some of our results about the Coulomb wave functions are also new. We shall discuss this in $\S 10$.

We now come to the remaining cases of (1.1) where $\ell \geq 3, k \leq 0$. It turns out that there does not appear to be a corresponding theory in the classical special functions that one could apply to this case of (1.1) as in the two special cases (1.10) and (1.25) where $\ell=1,2$, respectively. However, we shall show in $\S 9$, as the second purpose of this article, that finding a solution to the equation (1.1) that satisfies (1.3) when $\ell$ is odd is related to a classical theorem of Heine concerning the number of polynomial solutions of a second order differential equation. A third and final purpose of this article is to show (in $\S 10$ ) how new results (Theorems 10.1 and 10.2) concerning the finite-zero problems of Bessel functions and Coulomb wave functions can be derived as consequences of the methods used in proving the main theorems. These results have a bearing in several physical applications that we will briefly discuss in the $\S 10.5$.

This paper is organized as follows. The proof of Theorem 1.2 will be given in $\S 5$ after the discussion of the finite-zero problem of Bessel functions (1.16) in $\S 2$, the Lommel transformation for (1.15) in $\S 3$, and the Bessel polynomials in $\S 4$. The proofs of Theorems 1.3 and 1.6 are given in $\S 8$ after the discussion of the finite-zero problem of Coulomb wave functions (1.32) in $\S 6$ and the Lommel transformation for (1.20) in $\S 7$. However, the reader may skip $\S 2, \S 3, \S 4$ and $\S 6$ in the first reading. The statement of Theorem 9.1, its proof and the discussion of its relation to a problem of Heine will be discussed in $\S 9$. Finally, the theorems concerning the finite-zero problems of the Bessel and Coulomb wave functions will be stated and discussed in $\S 10.1$. Besides, several issues related to the main theme of this paper will also be discussed in the subsections in $\S 10$. Some frequently used formulae for the special functions are recorded in Appendix A.

We end this introduction by giving a number of remarks concerning the main theorems above and, in particular, we discuss briefly the connection of Theorem 1.3 and the subnormal solutions considered by Margrit Frei [21] and H. Wittich [43].

Remark 1.9 Suppose that $\ell>0$ and that $|k| \geq \ell$. If $f(z)$ is a solution to (1.1), then the function $g(z)=f(-z)$ satisfies (1.1) with $C(z)=A(-z)$. We notice that the corresponding $k$ and $\ell$ for $C(z)$ now satisfy $|k| \leq \ell$. If, however, $\ell<0$, the same transformation will convert the equation to an equation with coefficient $C(z)=$ $A(-z)$ but with the corresponding $\ell>0$. Thus it is sufficient to consider only the case when $\ell>0$ and $0 \leq|k| \leq \ell$ (since the case $0 \leq|\ell| \leq k$ can be transformed to the former). 
Remark 1.10 If both of the integers $k$ or $\ell$ in (1.2) are positive and at least one is odd, then Bank [3] and Gao [22] independently proved that any non-trivial solution of (1.1) must satisfy $\lambda(f)=+\infty$ (see also [4]). Hence it suffices to consider only the case when both $k$ and $\ell$ are even, or when $k=0$ and $\ell>0$ is odd.

Remark 1.11 The equation (1.17) can be transformed to the equation

$$
g^{\prime \prime}(z)+e^{-z} g^{\prime}(z)+K g(z)=0
$$

via the transformation $f(z)=g(z) \exp \left(-\frac{1}{2} e^{-z}\right)$. Frei [21] showed that the equation (1.33) admits a subnormal solution of the form $g(z)=e^{d z} S\left(e^{z}\right)$ if and only if $K=$ $-n^{2}$ for some integer $n$. Moreover, the polynomial $S(\zeta)$ mentioned above has degree $n$. An entire solution $g(z)$ to (1.33) is called subnormal if $\lim \sup _{r \rightarrow+\infty} \log T(r, g) / r=$ 0 . Thus, Bank and Laine's Theorem C generalized Frei's result. Wittich [43] (see also [24]) later also generalized Frei's result to a class of differential equations with coefficients that are polynomial in $e^{z}$. However, it appears that the connection of the equations (1.17) and (1.25) with Coulomb wave equations and the Bessel polynomials has been overlooked in the literature of complex differential equations. We also note that (1.17) was considered by P. M. Morse in 1929 in connection with wave mechanics problems.

\section{The Finite-Zero Problem of Bessel Functions}

We will show in this section that the condition for (1.11) to satisfy (1.3) is essentially equivalent to (1.16) having a finite number of zeros in the complex plane. To the best of the authors' knowledge, complete characterization of the finiteness of zeros of (1.16) for arbitrary (complex) $\nu$ was not previously known (see [15], [40, Chapter $\mathrm{XV}]$ ). The precise statement of this result will be given in Theorem 10.1. We shall first establish, in Lemma 2.1, that (1.16) has infinitely many zeros whenever $(A, B) \neq$ $(0,0)$. Since part of the proof of Theorem 10.1 uses Theorem 1.2, its proof can only be completed in $\S 10$. Our method also has the advantage that it shows pedagogically how we arrive at (1.13) from the general solution (1.11) when compared with the arguments used in [7] (for the general case (1.1)) and [8] (for the specific case (1.10)). Further comments concerning this problem will be given in $\S 10$.

We recall that the Bessel functions of the third kind of order $\nu$ or simply the Hankel functions [40, p. 73] are defined by

$$
H_{\nu}^{(1)}(x)=J_{\nu}(x)+i Y_{\nu}(x), \quad H_{\nu}^{(2)}(x)=J_{\nu}(x)-i Y_{\nu}(x) .
$$

Their asymptotics which we will use extensively here are given below by (A.1) and (A.2) in the Appendix.

Let $\delta$ be a positive number such that $\delta<\pi / 2$ and let $R, r$ be such that $R>r$. We define the semi-annular region

$$
\Omega(r, R ; \delta)=\left\{\rho e^{i \theta}: 0 \leq r \leq \rho \leq R ;-\pi+\delta \leq \theta \leq \pi-\delta\right\} .
$$


Clearly the complex plane cut from the origin to infinity along the negative real axis is given by

$$
\mathcal{C}:=\bigcup_{\delta>0} \Omega(0,+\infty ; \delta)
$$

Let $n_{\Omega}(r, R ; \delta)$ denote the number of zeros of a complex-valued function in the interior of $\Omega(r, R ; \delta)$. Then we define

$$
\lambda_{\delta}(r):=\limsup _{R \rightarrow+\infty} \frac{\log n_{\Omega}(r, R ; \delta)}{\log R}
$$

and

$$
\lambda^{*}:=\lim _{\delta \rightarrow 0} \lim _{r \rightarrow 0} \lambda_{\delta}(r)
$$

Lemma 2.1 Let $\nu, C$ and $D$ be three constants in $\mathbb{C}$ such that both $C$ and $D$ are nonzero. Then for each positive number $\delta, \delta<\pi / 2$, the combination of the Hankel functions $^{1}$

$$
U(\zeta)=C H_{\nu}^{(1)}(\zeta)+D H_{\nu}^{(2)}(\zeta)
$$

has an infinite number of zeros in $\Omega(0,+\infty ; \delta)$. Moreover,

$$
\lambda_{\delta}=: \limsup _{r \rightarrow 0} \lambda_{\delta}(r)=\limsup _{r \rightarrow 0} \limsup _{R \rightarrow+\infty} \frac{\log n(r, R ; \delta)}{\log R}=1 .
$$

In particular, we have

$$
\lambda^{*}=\limsup _{\delta \rightarrow 0} \lambda_{\delta}=1
$$

Proof Let $C$ and $D$ be two non-zero constants in (2.6) and let $\delta>0$ be given, $r$ and $R, r<R$, be chosen sufficiently large. Then both $H_{\nu}^{(1)}(\zeta)$ and $H_{\nu}^{(2)}(\zeta)$ are analytic in $\bar{\Omega}(r, R ; \delta)($ see $(2.2))$.

We shall show that the inequality

$$
\left|\left(\frac{\zeta \pi}{2}\right)^{\frac{1}{2}}\left(C H_{\nu}^{(1)}(\zeta)+D H_{\nu}^{(2)}(\zeta)\right)-\left(C e^{i X}+D e^{-i X}\right)\right|<\left|\left(C e^{i X}+D e^{-i X}\right)\right|
$$

where $X=\zeta-\frac{1}{2} \nu \pi-\frac{1}{4} \pi$, holds on the closure of $\Omega(r, R ; \delta)$ when $r, R$ are chosen to be suitably large and such that $0<r<R$.

\footnotetext{
${ }^{1}$ This refers to the principal branch.
} 
Let us write $\widetilde{C}=C e^{-i\left(\frac{1}{2} \nu \pi+\frac{1}{4} \pi\right)}$ and $\widetilde{D}=D e^{i\left(\frac{1}{2} \nu \pi+\frac{1}{4} \pi\right)}$, and $\zeta=r e^{i \theta}$. The asymptotic expansions (A.1) and (A.2), with $p=1$ in the remainders (A.3), give, when $R$ and $r$ $(R>r)$ are sufficiently large,

$$
\begin{aligned}
\mid\left(\frac{\zeta \pi}{2}\right)^{\frac{1}{2}}\left(C H_{\nu}^{(1)}(\zeta)\right. & \left.+D H_{\nu}^{(2)}(\zeta)\right)-\left(\widetilde{C} e^{i \zeta}+\widetilde{D} e^{-i \zeta}\right) \mid \\
& =\left|\widetilde{C} e^{i \zeta} R_{1}^{(1)}(\zeta)+\widetilde{D} e^{-i \zeta} R_{1}^{(2)}(\zeta)\right| \\
& \leq K\left[\left(\left|\widetilde{C} e^{i \zeta}\right|+\left|\widetilde{D} e^{-i \zeta}\right|\right)|\zeta|^{-1}\right] \\
& =K\left(|\widetilde{C}| e^{-r \sin \theta}+|\widetilde{D}| e^{r \sin \theta}\right) r^{-1}
\end{aligned}
$$

where $K$ is a positive constant. Thus for large $r$ the inequalities

$$
\begin{aligned}
\mid\left(\frac{\zeta \pi}{2}\right)^{\frac{1}{2}} & \left(C H_{\nu}^{(1)}(\zeta)+D H_{\nu}^{(2)}(\zeta)\right)-\left(\widetilde{C} e^{i \zeta}+\widetilde{D} e^{-i \zeta}\right) \mid \\
& \leq O\left(r^{-1}\right) \cdot \begin{cases}|\widetilde{D}| e^{r \sin \theta}\left(1+\frac{|\widetilde{C}|}{|\widetilde{D}|} e^{-2 r \sin \theta}\right) & \text { if } 0 \leq \theta \leq \pi-\delta, \\
|\widetilde{C}| e^{-r \sin \theta}\left(1+\frac{|\widetilde{D}|}{|\widetilde{C}|} e^{2 r \sin \theta}\right) & \text { if }-\pi+\delta \leq \theta<0,\end{cases} \\
& <\frac{\Delta_{1}}{r} e^{r|\sin \theta|}, \quad \text { if } 0 \leq|\theta| \leq \pi-\delta,
\end{aligned}
$$

hold, where $\Delta_{1}$ is a positive constant. On the other hand, we also have

$$
\begin{aligned}
\mid \widetilde{C} e^{i \zeta} & +\widetilde{D} e^{-i \zeta} \mid \\
& =\left|\left(\widetilde{C} e^{i r \cos \theta}\right) e^{-r \sin \theta}+\left(\widetilde{D} e^{-i r \cos \theta}\right) e^{r \sin \theta}\right| \\
& = \begin{cases}\left|\widetilde{D} e^{-i r \cos \theta} e^{r \sin \theta}\left(1+\frac{\widetilde{C}}{\widetilde{D}} \cdot e^{2 i r \cos \theta} e^{-2 r \sin \theta}\right)\right| & \text { if } 0 \leq \theta \leq \pi-\delta, \\
\left|\widetilde{C} e^{i r \cos \theta} e^{-r \sin \theta}\left(1+\frac{\widetilde{D}}{\widetilde{C}} \cdot e^{-2 i r \cos \theta} e^{2 r \sin \theta}\right)\right| & \text { if }-\pi+\delta \leq \theta<0 .\end{cases}
\end{aligned}
$$

We now define

$$
r_{p}=p \pi-\frac{1}{2} \arg \left(\frac{\widetilde{C}}{\widetilde{D}}\right),
$$

where the principal value of the argument is chosen. We set $r=|\zeta|=r_{p}$, where $p$ is a large positive integer.

Suppose first that $\theta=\arg \zeta$ satisfies

$$
0 \leq|\theta|<\left(\frac{\pi}{2 r_{p}}\right)^{\frac{1}{2}}
$$

Then

$$
2 r_{p}-\frac{\pi}{2}<2 r_{p}\left(1-\frac{\theta^{2}}{2}\right)<2 r_{p} \cos \theta<2 r_{p}
$$


Thus

$$
2 p \pi-\frac{\pi}{2}<\arg \left(\frac{\widetilde{C}}{\widetilde{D}} e^{2 i r \cos \theta}\right)<2 p \pi
$$

We deduce that

$$
\Re\left(\frac{\widetilde{C}}{\widetilde{D}} e^{2 i r \cos \theta} e^{-2 r \sin \theta}\right)>0 \text { and } \Re\left(\frac{\widetilde{D}}{\widetilde{C}} e^{-2 i r \cos \theta} e^{2 r \sin \theta}\right)>0 .
$$

Thus we obtain from (2.12) and (2.14), that if (2.13) holds, then

$$
\begin{aligned}
\left|\widetilde{C} e^{i r}+\widetilde{D} e^{-i r}\right| & \geq\left\{\begin{array}{l}
\left|\widetilde{D} e^{-i \zeta}\right| \quad \text { if } 0 \leq \theta<\left(\frac{\pi}{2 r_{p}}\right)^{\frac{1}{2}} \\
\left|\widetilde{C} e^{i \zeta}\right| \quad \text { if }-\left(\frac{\pi}{2 r_{p}}\right)^{\frac{1}{2}}<\theta<0
\end{array}\right. \\
& = \begin{cases}|\widetilde{D}| \max \left\{\left|e^{-i \zeta}\right|,\left|e^{i \zeta}\right|\right\}, \quad \text { if } 0 \leq \theta<\left(\frac{\pi}{2 r_{p}}\right)^{\frac{1}{2}}, \\
|\widetilde{C}| \max \left\{\left|e^{i \zeta}\right|,\left|e^{-i \zeta}\right|\right\}, \quad \text { if }-\left(\frac{\pi}{2 r_{p}}\right)^{\frac{1}{2}}<\theta<0\end{cases} \\
& \geq \Delta_{2} \max \left\{\left|e^{-i \zeta}\right|,\left|e^{i \zeta}\right|\right\} \quad \text { if } 0 \leq|\theta|<\left(\frac{\pi}{2 r_{p}}\right)^{\frac{1}{2}} \\
& =\Delta_{2} e^{r|\sin \theta|},
\end{aligned}
$$

if (2.13) holds where $\Delta_{2}=\min \{|\widetilde{C}|,|\widetilde{D}|\}$.

Suppose next that (2.13) is false, so that

$$
\left(\frac{\pi}{2 r_{p}}\right)^{\frac{1}{2}} \leq|\theta| \leq \pi-\delta
$$

We now choose the integer $p$ in $r_{p}$ so large that

$$
\left(\frac{\pi}{2 r_{p}}\right)^{\frac{1}{2}} \leq \delta
$$

Then (2.16) yields

$$
\left|2 r_{p} \sin \theta\right| \geq 2 r_{p} \sin \left(\frac{\pi}{2 r_{p}}\right)^{\frac{1}{2}} \geq 2 r_{p} \frac{2}{\pi}\left(\frac{\pi}{2 r_{p}}\right)^{\frac{1}{2}}=\left(\frac{8 r_{p}}{\pi}\right)^{\frac{1}{2}} .
$$

Thus for $\theta$ lies in the range (2.16) and if $p$ is large, we deduce from (2.12) that (2.17)

$$
\begin{aligned}
\mid \widetilde{C} e^{i \zeta} & +\widetilde{D} e^{-i \zeta} \mid \\
& \geq \begin{cases}\left|\widetilde{D} e^{-i r \cos \theta} e^{r \sin \theta}\right|\left(1-|\widetilde{C}| \widetilde{D} \mid e^{-\left(8 r_{p} / \pi\right)^{\frac{1}{2}}}\right) & \text { if }\left(\frac{\pi}{2 r_{p}}\right)^{\frac{1}{2}} \leq \theta \leq \pi-\delta, \\
\left|\widetilde{C} e^{i r \cos \theta} e^{-r \sin \theta}\right|\left(1-\left|\frac{\widetilde{D}}{\widetilde{C}}\right| e^{-\left(8 r_{p} / \pi\right)^{\frac{1}{2}}}\right) & \text { if }-\pi+\delta \leq \theta \leq-\left(\frac{\pi}{2 r_{p}}\right)^{\frac{1}{2}}\end{cases} \\
& \geq \frac{\Delta_{2}}{2} e^{r|\sin \theta|} .
\end{aligned}
$$


We deduce immediately from (2.15) that (2.17) holds for $|\zeta|=r_{p}$ and $|\arg \zeta| \leq$ $\pi-\delta$. We now choose large integers $p$ and $q$ such that $q>p$ and consider $\Omega\left(r_{p}, r_{q} ; \delta\right)$. Clearly both the inequalities (2.11) and (2.17) hold simultaneously on the two circular arcs and two straight line segments of $\Omega\left(r_{p}, r_{q} ; \delta\right)$. We conclude that the inequality (2.9) holds on all the boundary points of $\Omega\left(r_{p}, r_{q} ; \delta\right)$.

We now apply Rouché's theorem to the inequality (2.9) to conclude that the function

$$
\left(\frac{\zeta \pi}{2}\right)^{\frac{1}{2}}\left[C H_{\nu}^{(1)}(\zeta)+D H_{\nu}^{(2)}(\zeta)\right]
$$

has equally many zeros as the function

$$
C e^{i X}+D e^{-i X}
$$

in the interior of $\Omega\left(r_{p}, r_{q} ; \delta\right)$. But since both of $C$ and $D$ are not equal to zero, so the zeros of (2.18) are given by, for all $k$ sufficiently large,

$$
\zeta=\frac{1}{2} \pi \nu+\frac{1}{4} \pi+\frac{1}{2 i}\left[\log \left(-\frac{D}{C}\right)+2 \pi k i\right] .
$$

Thus we deduce that the number of zeros in $\Omega\left(r_{p}, r_{q} ; \delta\right)$ as $q \rightarrow \infty$ is

$$
\frac{2 r_{q}}{\pi}+O(1)
$$

for a fixed $p$. The conclusion of course remains true when $r_{p}=r \rightarrow 0$. This gives (2.7).

Clearly the same inequality is valid for each $\delta>0$. We complete the proof by letting $\delta \rightarrow 0$. This establishes (2.8).

Lemma 2.2 Let $\mathcal{c}, C, D$ and $\nu$ be members of $\mathbb{C}$, and such that the first three constants are non-zero. Then the entire function

$$
J(z)=C H_{\nu}^{(1)}\left(c e^{z}\right)+D H_{\nu}^{(2)}\left(c e^{z}\right)
$$

has an infinite exponent of convergence of zeros.

Proof For each sufficiently small $\delta>0$ and $r>0$, it follows from (2.7) of Lemma 2.1 that (2.6) has infinitely many zeros in $\Omega(r,+\infty ; \delta)$.

It follows from (2.7) that given any $0<\varepsilon<1$ there exists a (infinite) sequence $\left\{\zeta_{n}\right\}$ of zeros of (2.6) with $\left|\zeta_{n}\right|=\rho_{n}$ in $\Omega(r,+\infty ; \delta)$ such that

$$
n\left(r, \rho_{n} ; \delta\right) \geq \rho_{n}^{1-\varepsilon}
$$

for all $n$ sufficiently large.

Without loss of generality, we may choose $c=1$. We now make the substitution $e^{z}=\zeta$, where $z=r e^{i \theta}$ and $\zeta=\rho e^{i \phi}$. We define $U(\zeta)$ so that $J(z)=U\left(c e^{z}\right)$. Let 
$n(\mathcal{D}, F)$ denote the number of zeros of a function $F$ in $\mathcal{D}$. Then we have, by writing $e^{z_{n}}=\zeta_{n}, z_{n}=r_{n} e^{\theta_{n}}, e^{r_{n}}=\rho_{n}$, and with the above inequalities, that

$$
\begin{aligned}
& \frac{\log n\left\{|z| \leq \log \rho_{n}+\pi, J(z)\right\}}{\log r_{n}} \\
& \geq \frac{\log n\left\{\left[1 / \rho_{n} \leq|\zeta| \leq \rho_{n}\right], U(\zeta)\right\}}{\log r_{n}} \\
& \geq \frac{\log \left(\rho_{n}\right)^{1-\varepsilon}}{\log r_{n}}=\frac{(1-\varepsilon) \log \rho_{n}}{\log \log \rho_{n}},
\end{aligned}
$$

which tends to infinity as $n \rightarrow+\infty$. If $c$ is an arbitrary non-zero number, then we have $c e^{z}=\zeta$, or $e^{z}=\zeta / c$. Hence the above argument again holds after we replace $\zeta$ by $\zeta / c$.

\section{Lommel Transformations}

Lommel [35] and Pearson [36] independently (see also [40, p. 97]) studied the following transformation given by

$$
x=\alpha t^{\beta}, \quad y(x)=t^{\gamma} u(t),
$$

where $\alpha, \beta$ and $\gamma$ are constants and applied to the Bessel equation (1.15). We note that we have slightly adjusted the transformation above in comparison with those in [40, p. 97]. The application of the Lommel transformation leads to differential equations of different forms whose solutions are related to Bessel functions. We shall consider the following generalized Bessel equation

$$
x^{2} y^{\prime \prime}+x y^{\prime}+\left(\sum_{j=-n^{\prime}}^{n} k_{j} x^{j}\right) y=0 .
$$

Compute $y^{\prime}$ and $y^{\prime \prime}$ by (3.1) and substitute them into (3.2) to get

$$
t^{2} u^{\prime \prime}(t)+(2 \gamma+1) t u^{\prime}(t)+\left(\gamma^{2}+\beta^{2} \sum_{j=-n^{\prime}}^{n} \alpha^{j} k_{j} t^{\beta j}\right) u(t)=0
$$

A further change of variables by

$$
t=e^{m z}, \quad f(z)=u(t)
$$

leads to an equation of the form

$$
f^{\prime \prime}+2 \gamma m f^{\prime}+\left(\gamma^{2}+\beta^{2} \sum_{j=-n^{\prime}}^{n} \alpha^{j} k_{j} e^{\beta m j z}\right) f=0
$$


In the case of the Bessel equation (1.15), equation (3.3) becomes

$$
t^{2} u^{\prime \prime}(t)+(2 \gamma+1) t u^{\prime}(t)+\left(\alpha^{2} \beta^{2} t^{2 \beta}+\gamma^{2}-\nu^{2} \beta^{2}\right) u(t)=0
$$

The general solution to (3.6) is thus given by

$$
u(t)=t^{-\gamma}\left[A J_{\nu}\left(\alpha t^{\beta}\right)+B Y_{\nu}\left(\alpha t^{\beta}\right)\right],
$$

where $A$ and $B$ are arbitrary constants. The substitution of (3.4) in (3.5), where $m$ is an integer, yields

$$
f^{\prime \prime}(z)+2 \gamma m f^{\prime}(z)+\left(\alpha^{2} \beta^{2} e^{2 m \beta z}+\gamma^{2}-\nu^{2} \beta^{2}\right) f(z)=0
$$

and the solution of (3.8) becomes

$$
f(z)=e^{-\gamma z}\left[A J_{\nu}\left(\alpha e^{\beta m z}\right)+B Y_{\nu}\left(\alpha e^{\beta m z}\right)\right]
$$

(see also [40, p. 99]). We shall apply the Lommel transformation to the Coulomb wave equations in relation to the study of equations (1.17) and (1.25) in $\S 7$.

\section{The Bessel Polynomials}

We now collect some facts about the Bessel polynomials to be used in the sequel. The reader may choose to skip this section in the first reading. Our main reference is the original paper of Krall and Frink [33] and Grosswald's classic [23].

Lemma 4.1 For each positive integer $n$, the differential equation

$$
\zeta w^{\prime \prime}(\zeta)-2(\zeta+n) w^{\prime}(\zeta)+2 n w(\zeta)=0
$$

admits a polynomial solution of degree $n$, given by

$$
\theta_{n}(\zeta)=\sum_{j=0}^{n} a_{j}^{(n)} \zeta^{n-j}, \quad a_{j}^{(n)}=\frac{(n+j) !}{2^{n}(n-j) ! j !}
$$

Moreover, the equation with $n$ replaced by $-n$ has a rational solution $\theta_{-n}(\zeta)$ given by

$$
\theta_{-n}(\zeta):=\zeta^{-2 n+1} \theta_{n-1}(\zeta), \quad \text { or } \quad \theta_{n}(\zeta):=\zeta^{2 n+1} \theta_{-(n+1)}(\zeta)
$$

Furthermore the polynomial

$$
y_{n}(\zeta)=\zeta^{n} \theta_{n}(1 / \zeta)
$$

satisfies the equation

$$
\zeta^{2} y^{\prime \prime}(\zeta)+2(\zeta+1) y^{\prime}(\zeta)-n(n+1) y(\zeta)=0
$$


The reader can easily verify Lemma 4.1. A proof is given in [23, p. 8].

Following Grosswald [23], we call $y_{n}$ the Bessel polynomial of degree $n$ and $\theta_{n}(x)$ the reverse Bessel polynomial of degree $n$. We record here formulae that relate Bessel polynomials and Bessel functions of half of an integer order:

$$
J_{n+\frac{1}{2}}(x)=\left(\frac{2}{\pi x}\right)^{\frac{1}{2}}\left(\frac{1}{i^{n+1}} e^{i x} y_{n}\left(-\frac{1}{i x}\right)+i^{n+1} e^{-i x} y_{n}\left(\frac{1}{i x}\right)\right),
$$

and

$$
J_{-n-\frac{1}{2}}(x)=\left(\frac{2}{\pi x}\right)^{\frac{1}{2}}\left(i^{n} e^{i x} y_{n}\left(-\frac{1}{i x}\right)+i^{-n} e^{-i x} y_{n}\left(\frac{1}{i x}\right)\right) .
$$

Krall and Frink also studied the generalized Bessel polynomials [33] which are solutions to the differential equation

$$
\zeta^{2} y^{\prime \prime}(\zeta)+(a \zeta+b) y^{\prime}(\zeta)-n(n+a-1) y(\zeta)=0,
$$

where $n$ is a non-negative integer. Clearly the equation (4.8) reduces to (4.5) when $a=b=2$. The polynomial solution to (4.8) is given by

$$
y_{n}(\zeta ; a, b)=\sum_{k=0}^{n}\left(\begin{array}{l}
n \\
k
\end{array}\right)(n+k+a-2)_{k}\left(\frac{\zeta}{b}\right)^{k},
$$

for $n=0,1,2, \ldots$, where

$$
(a)_{0}=1, \quad(a)_{k}=a(a+1) \cdots(a+k-1),
$$

are the shifted factorials (see [2, p. 2]).

Remark 4.2 If $a=0,-1,-2, \ldots$, in (4.9), then $y_{n}(\zeta ; a, b)$ may have degree less than $n$.

\section{Proof of Theorem 1.2}

Suppose that the equation (1.10) admits a non-trivial solution $y(z)$. Then by the Lommel transformation in (3.1) and by comparing the coefficients between the equations (3.8) and (1.10), we deduce that $m=1, \alpha=2 \sqrt{K_{1}}, \beta=1 / 2$ and $\gamma=0$. Hence it follows from (3.7) and (3.4) that the general solution of (1.10) can be written in the form

$$
y(z)=u\left(e^{z}\right)=G\left(e^{z / 2}\right), \quad G(\zeta)=A J_{\nu}\left(2 \sqrt{K_{1}} \zeta\right)+B Y_{\nu}\left(2 \sqrt{K_{1}} \zeta\right) .
$$

This gives (1.11). Since $f$ has the form (5.1), the assumption (1.3) must hold independently of the particular branch of the Bessel functions chosen. That is, the result (1.3) must hold for each branch of the function $G(\zeta)$. It remains to find the desired form of $f(z)$ when it satisfies (1.3). It is easily seen from (1.11) that all solutions of 
(1.10) are entire functions. Note that we may rewrite $G(\zeta)$ in terms of the Hankel functions:

$$
G(\zeta)=C H_{\nu}^{(1)}\left(2 \sqrt{K_{1}} \zeta\right)+D H_{\nu}^{(2)}\left(2 \sqrt{K_{1}} \zeta\right)
$$

where

$$
C=(A-i B) / 2, \quad D=(A+i B) / 2,
$$

and, without loss of generality, to assume that the $H_{\nu}^{(1),(2)}(\zeta)$ are their principal branches. It follows from (2.1) that the $G(\zeta)$ is analytic in the region $\mathcal{C}$ defined in (2.3). If $C D \neq 0$, then Lemma 2.1 shows that the function $G(\zeta)$ has infinitely many zeros in C. In particular, (2.8) holds. We see immediately from Lemmas 2.1 and 2.2 that the existence of a non-constant $f(z)$ that satisfies (1.3) implies that $G(\zeta)$ has a finite number of zeros, and hence exactly one of $C$ or $D$ is equal to zero. Without loss of generality, we may assume that $D=0$ and $C=1$ in (5.2). That is, we have $f(z)=$ $H_{\nu}^{(1)}\left(2 \sqrt{K_{1}} e^{z / 2}\right)$. On the other hand, the condition $\lambda(f)=\lambda\left(H_{\nu}^{(1)}\left(2 \sqrt{K_{1}} e^{z / 2}\right)\right)<$ $+\infty$ must hold independently in each branch of the $H_{\nu}^{(1)}(\zeta)$.

We recall that the analytic continuation of the Hankel function is given by

$$
H_{\nu}^{(1)}\left(z e^{m \pi i}\right):=\frac{\sin (1-m) \nu \pi}{\sin \nu \pi} H_{\nu}^{(1)}(z)-e^{-\nu \pi i} \frac{\sin m \nu \pi}{\sin \nu \pi} H_{\nu}^{(2)}(z),
$$

where $m$ is any integer (see [40, p. 75]). The right-hand side of (5.4) is the principal branch of the Hankel functions. We shall choose $m$ to be an even integer in what follows. Thus, without loss of generality, we may assume that

$$
\begin{aligned}
f(z) & =H_{\nu}^{(1)}\left(2 \sqrt{K_{1}} e^{z / 2}\right) \\
& =H_{\nu}^{(1)}\left(2 \sqrt{K_{1}} e^{z / 2} e^{i m \pi}\right) \\
& =\widehat{C} H_{\nu}^{(1)}\left(2 \sqrt{K_{1}} e^{z / 2}\right)+\widehat{D} H_{\nu}^{(2)}\left(2 \sqrt{K_{1}} e^{z / 2}\right)
\end{aligned}
$$

must hold for each non-zero even integer $m$, where

$$
\widehat{C}=\frac{\sin (1-m) \nu \pi}{\sin \nu \pi} \text { and } \widehat{D}=-e^{-\nu \pi i} \frac{\sin m \nu \pi}{\sin \nu \pi} .
$$

Since the right-hand side of (5.4) is the principal branch, so Lemma 2.2 implies that at least one of the coefficients $\widehat{C}$ or $\widehat{D}$ in (5.6) must be zero in order for $f(z)$ to satisfy (1.3). It is straightforward to check that each of the constants $\widehat{C}$ and $\widehat{D}$ in (5.6) has finite non-zero limit as $\nu \rightarrow l$ for any non-zero integer $l$. So when $\nu$ is a non-zero integer, Lemma 2.1 implies that (5.4) has infinitely many zeros. Thus, we may exclude the case when $\nu$ equals an integer. Let us assume henceforth that $\nu$ is not an integer. Suppose that the constant $\widehat{C}$ equals zero. Since (5.2) is independent of the branches, the analytic continuation principle and the identity theorem indicate that we must look for a single $\nu$ at which the constant $\widehat{C}=0$ for all branches of $H_{\nu}^{(1)}(\zeta)$. That is, we seek 
a single $\nu$ for which $\sin (1-m) \nu \pi=0$ for each even $m$. Let $m=2 s$ for some integer $s$. That is, $\nu=k /(2 s-1), s, k \in \mathbb{Z}$ and that $\nu \notin \mathbb{Z}$. By the identity theorem, the parameter $\nu$ that we are looking for must appear in the formula for all the choices of even $m$. This is equivalent to the case when $s=1$, that is, when $\nu$ is an integer. But this case was already excluded in the above consideration. Thus, there does not exist a single $\nu$ for which $\widehat{C}=0$ for all branches of (5.4), a contradiction. So $\widehat{C}$ cannot be zero.

Since $\widehat{C} \neq 0$ if (5.5) satisfies (1.3), then Lemma 2.1 implies that it remains to consider $\widehat{D}=0$. That is, $\sin m \nu \pi=0$. By the analytic continuation principle and the identity theorem, we again look for a single $\nu$ for which $\widehat{D}=0$ for each branch of $H_{\nu}^{(1)}(\zeta)$. So we write $m=2 s$ for some non-zero integer $s$. Thus, $2 s \nu \pi=k \pi$ for $s \in \mathbb{Z}$ and $k \in \mathbb{Z}$. That is, except when both sides are zero, we have $\nu=k / 2 s(s \in \mathbb{N}$ and $k \in \mathbb{Z}$ ). We recall that $\nu$ is not an integer. By the identity theorem again, the $\nu$ that we are looking for must appear in the above formula for all the possible $s$ and $k$ except when $\nu$ is an integer. A careful, but otherwise straightforward analysis shows that this is equivalent to the case $s=1$, or equivalently when $k$ is odd, i.e., when $\nu=n+\frac{1}{2}$ for some $n \in \mathbb{Z}$. This proves that if any branch of $H_{\nu}^{(1)}(\zeta)$, except perhaps the principal branch, is to have a finite number of zeros in $-\pi+2 \pi k<\arg \zeta<$ $\pi+2 \pi k, k= \pm 1, \pm 2, \ldots$, then $\nu=n+\frac{1}{2}$ for some $n \in \mathbb{Z}$. It remains to consider the principal branch of $H_{\nu}^{(1)}(\zeta)$. But if it has a finite number of zeros in the region $\mathcal{C}$, then by the identity theorem, its $\nu$ must be amongst the numbers $\nu=n+\frac{1}{2}$. However, it is known that $H_{\nu}^{(1)}(\zeta)$ has a finite number of zeros when $\nu=n+\frac{1}{2}$ for each $n \in \mathbb{Z}$ (see $[18$, p. 10]), so this shows that for each branch of $(1.16)$ to have a finite number of zeros, we must have $\nu=n+\frac{1}{2}$ for some $n \in \mathbb{Z}$.

Now since $D=0$ and $\nu=n+1 / 2$, we deduce from (5.3) that $B=-i A$. We further assume that $n \geq 0$. It follows from the definition [40, p. 64] that $Y_{n+\frac{1}{2}}=$ $(-1)^{n+1} J_{-\left(n+\frac{1}{2}\right)}$. Thus,

$$
\begin{aligned}
G(\zeta) & =A\left[J_{n+\frac{1}{2}}\left( \pm 2 \sqrt{K_{1}} \zeta\right)+i Y_{n+\frac{1}{2}}\left( \pm 2 \sqrt{K_{1}} \zeta\right)\right] \\
& =A\left[J_{n+\frac{1}{2}}\left( \pm 2 \sqrt{K_{1}} \zeta\right)+(-1)^{n+1} i J_{-\left(n+\frac{1}{2}\right)}\left( \pm 2 \sqrt{K_{1}} \zeta\right)\right]
\end{aligned}
$$

In order to illustrate the underlying principle of how the solution can have only a finite number of zeros, we have taken a pedagogically more natural, but longer, approach in the following argument. ${ }^{2}$

Let us first consider the case when the argument in $J_{\nu}$ is + . We deduce from (5.2), (4.6) and (4.7) that for both even and odd $n$,

$$
\begin{aligned}
G_{+}(\zeta) & =A\left[J_{n+\frac{1}{2}}\left(2 \sqrt{K_{1}} \zeta\right)+i Y_{n+\frac{1}{2}}\left(2 \sqrt{K_{1}} \zeta\right)\right] \\
& =A\left[J_{n+\frac{1}{2}}\left(2 \sqrt{K_{1}} \zeta\right)+(-1)^{n+1} i J_{-\left(n+\frac{1}{2}\right)}\left(2 \sqrt{K_{1}} \zeta\right)\right]
\end{aligned}
$$

\footnotetext{
${ }^{2}$ We note that one can connect (5.7) to the Bessel polynomials via a modified Bessel function directly.
} 


$$
\begin{gathered}
=A\left[( \frac { 1 } { \pi \sqrt { K _ { 1 } } \zeta } ) ^ { 1 / 2 } \left(\frac{1}{i^{n+1}} e^{+2 i \sqrt{K_{1}} \zeta} y_{n}\left(-\frac{1}{2 i \sqrt{K_{1}} \zeta}\right)\right.\right. \\
\left.+i^{n+1} e^{-2 i \sqrt{K_{1}} \zeta} y_{n}\left(\frac{1}{2 i \sqrt{K_{1}} \zeta}\right)\right) \\
+(-1)^{n+1}\left(\frac{1}{\pi \sqrt{K_{1}} \zeta}\right)^{1 / 2}\left(i^{n+1} e^{+2 i \sqrt{K_{1}} \zeta} y_{n}\left(-\frac{1}{2 i \sqrt{K_{1}} \zeta}\right)\right. \\
=A\left(\frac{1}{\pi \sqrt{K_{1}} \zeta}\right)^{1 / 2}\left[e^{+2 i \sqrt{K_{1}} \zeta}\left(\frac{1}{i^{n+1}}+(-1)^{n+1} i^{n+1}\right) y_{n}\left(-\frac{1}{2 i \sqrt{K_{1}} \zeta}\right)\right. \\
\left.=A\left(\frac{1}{\pi \sqrt{K_{1}} \zeta}\right)^{1 / 2} \frac{2}{i^{n+1}} e^{+2 i \sqrt{K_{1}} \zeta} y_{n}\left(-\frac{1}{2 i \sqrt{K_{1}} \zeta}\right)\right]
\end{gathered}
$$

But $G_{+}(\zeta)=u\left(\zeta^{2}\right)$. So

$$
u(\zeta)=A\left(\frac{1}{\pi \sqrt{K_{1}} \zeta^{1 / 2}}\right)^{1 / 2} \frac{2}{i^{n+1}} e^{+2 i \sqrt{K_{1}} \zeta^{1 / 2}} y_{n}\left(\frac{-1}{2 i \sqrt{K_{1}} \zeta^{1 / 2}}\right)
$$

Hence

$$
y_{+}(z)=u\left(e^{z}\right)=A \frac{2}{i^{n+1}}\left(\frac{1}{\pi \sqrt{K_{1}}}\right)^{1 / 2} e^{-z / 4} y_{n}\left(\frac{-1}{2 i \sqrt{K_{1}}} e^{-z / 2}\right) \exp \left(+2 i \sqrt{K_{1}} e^{z / 2}\right) .
$$

We now write $f(z)$ in terms of the reverse Bessel polynomials. Applying (4.4) to $y_{+}$gives:

$$
\begin{aligned}
y_{+}(z)= & A \frac{2}{i^{n+1}}\left(\frac{1}{\pi \sqrt{K_{1}}}\right)^{1 / 2} e^{-z / 4}\left(\frac{-1}{2 i \sqrt{K_{1}}} e^{-z / 2}\right)^{n} \theta_{n}\left(-2 i \sqrt{K_{1}} e^{z / 2}\right) \\
& \times \exp \left(+2 i \sqrt{K_{1}} e^{z / 2}\right) \\
= & \widetilde{A} \frac{2}{i^{n+1}}\left(\frac{1}{\pi \sqrt{K_{1}}}\right)^{1 / 2} e^{d z}(-1)^{n} \theta_{n}\left(-2 i \sqrt{K_{1}} e^{z / 2}\right) \exp \left(+2 i \sqrt{K_{1}} e^{z / 2}\right)
\end{aligned}
$$

where $d=-(2 n+1) / 4$ and $K_{0}=(\beta \nu)^{2}=\left(\frac{1}{2}\left(n+\frac{1}{2}\right)\right)^{2}=(2 n+1)^{2} / 16$. We now consider the second case where the argument of $J_{\nu}$ is - . We have

$$
\begin{aligned}
G_{-}(\zeta) & =A\left[J_{n+\frac{1}{2}}\left(-2 \sqrt{K_{1}} \zeta\right)+i Y_{n+\frac{1}{2}}\left(-2 \sqrt{K_{1}} \zeta\right)\right] \\
& =A\left[J_{n+\frac{1}{2}}\left(-2 \sqrt{K_{1}} \zeta\right)+(-1)^{n+1} i J_{-\left(n+\frac{1}{2}\right)}\left(-2 \sqrt{K_{1}} \zeta\right)\right]
\end{aligned}
$$

The remaining steps for $y_{-}$are essentially the same as the $y_{+}$case except we need to replace the + signs by - signs in $y_{n}, \theta_{n}$ and in the exponential factors, so we omit the details. This gives (1.13) and proves the case when $n$ is non-negative. 
Suppose that $n=-m$ where $m$ is positive, then we replace $n$ in all the above steps by $-m$. Applying the formula (4.3) to (5.8) yields

$$
\begin{aligned}
y_{ \pm}(z) & =\theta_{n}\left(\mp 2 i \sqrt{K_{1}} e^{z / 2}\right) \exp \left( \pm 2 i \sqrt{K_{1}} e^{z / 2}+d z\right) \\
& =\theta_{-m}\left(\mp 2 i \sqrt{K_{1}} e^{z / 2}\right) \exp \left( \pm 2 i \sqrt{K_{1}} e^{z / 2}+d z\right) \\
& =(\text { const }) \theta_{m-1}\left(\mp 2 i \sqrt{K_{1}} e^{z / 2}\right)\left(e^{z / 2}\right)^{-2 m+1} \exp \left( \pm 2 i \sqrt{K_{1}} e^{z / 2}+d z\right) \\
& =(\text { const }) \theta_{m-1}\left(\mp 2 i \sqrt{K_{1}} e^{z / 2}\right) \exp \left( \pm 2 i \sqrt{K_{1}} e^{z / 2}+d^{\prime} z\right)
\end{aligned}
$$

where

$$
d^{\prime}=\frac{-2 m+1}{2}+\frac{-2 n-1}{4}=\frac{-2 m+1}{2}+\frac{2 m-1}{4}=\frac{-2 m+1}{4},
$$

as asserted. We note that we can obtain $d^{\prime}$ directly by replacing $n$ by $m-1$ in (1.14).

To prove the converse part, we define $f$ by (5.8) or (5.10) with the constants chosen as above. The function $f$ can be verified to satisfy equation (1.10) with $K_{0}$ given by (1.12), and $f$ has a finite exponent of convergence of zeros. This completes the proof.

Remark 5.1 We note that the integer $n$ in (1.7) in the statement of Theorem B is only allowed to be non-negative. We can allow for negative $n$ in (1.12) of Theorem 1.2 in the above argument due to the application of (4.3), (4.6) and (4.7).

Remark 5.2 The above argument also proves that for each branch of (5.4) to have a finite number of zeros in $\zeta$ in

$$
-\pi+2 \pi k<\arg \zeta<\pi+2 \pi k, \quad k=0, \pm 1, \pm 2, \ldots
$$

then it reduces to

$$
\begin{aligned}
H_{n+\frac{1}{2}}^{(1)}\left(\zeta e^{2 s \pi i}\right) & =\frac{\sin (1-2 s)\left(n+\frac{1}{2}\right) \pi}{\sin \left(n+\frac{1}{2}\right) \pi} H_{n+\frac{1}{2}}^{(1)}(\zeta)-e^{-\left(n+\frac{1}{2}\right) \pi i} \frac{\sin 2 s\left(n+\frac{1}{2}\right) \pi}{\sin \left(n+\frac{1}{2}\right) \pi} H_{n+\frac{1}{2}}^{(2)}(\zeta) \\
& = \pm H_{n+\frac{1}{2}}^{(1)}(\zeta),
\end{aligned}
$$

where $s \in \mathbb{Z}$.

\section{The Finite-Zero Problem of Coulomb Wave Functions}

The first of two linearly independent solutions of the Coulomb wave equation (1.20) is given by the regular Coulomb wave function defined as

$$
F_{L}(\eta, x)=C_{L}(\eta) x^{L+1} e^{-i x} M(L+1-i \eta, 2 L+2,2 i x),
$$


where $C_{L}(\eta)$ is the Gamow factor ${ }^{3}$ defined by [39, p. 494]

$$
C_{L}(\eta)=2^{L} e^{i \sigma_{L}(\eta)-\frac{\pi \eta}{2}} \frac{\Gamma(L+1-i \eta)}{\Gamma(2 L+2)},
$$

where $L$ and $\eta$ are complex constants, and $M(a, b, z)$ is a confluent hypergeometric (entire) function [1, p. 504], and it is a solution to the Kummer equation [1, 2, 11, 42]. A second linearly independent solution to (1.20) is given by the irregular Coulomb wave function defined as

$$
G_{L}(\eta, x)= \begin{cases}\frac{F_{-L-1}(\eta, x)-\cos x(L, \eta) F_{L}(\eta, x)}{\sin x(L, \eta)} & \text { if } 2 L \text { is not an integer } \\ \frac{\frac{\partial F_{-L-1}(\eta, x)}{\partial L}-\cos x(L, \eta) \frac{\partial F_{-L-1}(\eta, x)}{\partial L}}{\cos x(L, \eta) \frac{\partial x(L \eta)}{\partial L}} & \text { if } 2 L \text { is an integer }\end{cases}
$$

where the phase function $x(L, \eta)[17, \S 6]$ is given in (A.6).

We further introduce the modified Coulomb wave functions (see [17]):

$$
\psi_{ \pm}(L, \eta, x)=G_{L}(\eta, x) \pm i F_{L}(\eta, x)
$$

in analogy to the Bessel functions of the third kind (Hankel functions). We have the following analogue of Lemma 2.1.

Lemma 6.1 Let $C$ and $D$ be two non-zero constants. Then, for each positive number $\delta$, $0<\delta<\pi / 2$, the combination of the modified Coulomb wave functions ${ }^{4}$

$$
V(\zeta)=C \psi_{+}(L, \eta ; \zeta)+D \psi_{-}(L, \eta ; \zeta)
$$

has an infinite number of zeros in $\Omega(0,+\infty ; \delta)$. In particular, (2.7) and (2.8) hold for $V(\zeta)$

Proof We assume both $C$ and $D$ to be non-zero constants. Let $\delta>0$ be fixed. Then both $\psi_{+}$and $\psi_{-}$are analytic in $\Omega(r, R ; \delta)$. Suppose $(6.4)$ has only a finite number of zeros in $\Omega(0,+\infty ; \delta)$. Let

$$
\chi:=\chi(L, \eta ; \zeta)=\zeta-\eta \log (2 \zeta)-L \pi / 2+\sigma_{L}(\eta)
$$

We shall show that the inequality

$$
\begin{aligned}
\mid\left(C \psi_{+}(L, \eta ; \zeta)+D \psi_{-}(L, \eta ; \zeta)\right)-\left(C e^{i \chi(L, \eta ; \zeta)}\right. & \left.+D e^{-i \chi(L, \eta ; \zeta)}\right) \mid \\
& <\left|C e^{i \chi(L, \eta ; \zeta)}+D e^{-i \chi(L, \eta ; \zeta)}\right|
\end{aligned}
$$

holds on the closure of $\Omega(r, R ; \delta)$.

\footnotetext{
${ }^{3}$ This factor is more general than the one given in [1, p. 537] since we consider complex $L$ and $\eta$.

${ }^{4}$ This refers to their principal branch.
} 
Let $\widehat{C}=C e^{-i\left(L \pi / 2+\sigma_{L}(\eta)+\eta \log 2\right)}$, and $\widehat{D}=D e^{i\left(L \pi / 2-\sigma_{L}(\eta)+\eta \log 2\right)}$ and $\zeta=r e^{i \theta}$. We further let $\eta=\eta_{1}+i \eta_{2}$,

$$
\begin{aligned}
& \chi_{1}=r \cos \theta-\eta_{1} \log r+\eta_{2} \theta, \\
& \chi_{2}=r \sin \theta-\eta_{2} \log r-\eta_{1} \theta,
\end{aligned}
$$

where $\eta_{1}, \eta_{2}, \chi_{1}$ and $\chi_{2}$ are all real.

Let $R$ and $r(R>r)$ be sufficiently large. Then the asymptotic expansions (A.7) and (A.8) of the modified Coulomb wave functions (6.3) with the remainders (A.9) with $p=1$ imply

$$
\begin{aligned}
\mid\left(C \psi_{+}(L, \eta ; \zeta)\right. & \left.+D \psi_{-}(L, \eta ; \zeta)\right)-\left(C e^{i \chi(L, \eta ; \zeta)}+D e^{-i \chi(L, \eta ; \zeta)}\right) \mid \\
& =K\left|C e^{i \chi(L, \eta ; \zeta)} L_{1}^{(+)}(\zeta)+D e^{-i \chi(L, \eta ; \zeta)} L_{1}^{(-)}(\zeta)\right| \\
& \leq K\left[\left(\left|C e^{i \chi(L, \eta ; \zeta)}\right|+\left|D e^{-i \chi(L, \eta ; \zeta)}\right|\right)|\zeta|^{-1}\right] \\
& =K\left[\left(\left|\widehat{C} e^{i\left(\chi_{1}+i \chi_{2}\right)}\right|+\left|\widehat{D} e^{-i\left(\chi_{1}+i \chi_{2}\right)}\right|\right)|\zeta|^{-1}\right] \\
& =K\left[\left(|\widehat{C}| e^{-\chi_{2}}+|\widehat{D}| e^{\chi_{2}}\right)|\zeta|^{-1}\right]
\end{aligned}
$$

holds on the boundary and in the interior of $\Omega(r, R ; \delta)$. Here the $K$ denotes a positive constant.

Thus for large $r$ we have the inequalities

$$
\begin{aligned}
\mid\left(C \psi_{+}(L, \eta ; \zeta)\right. & \left.+D \psi_{-}(L, \eta ; \zeta)\right)-\left(C e^{i \chi(L, \eta ; \zeta)}+D e^{-i \chi(L, \eta ; \zeta)}\right) \mid \\
& =O\left(\frac{1}{r}\right) \cdot\left\{\begin{array}{l}
|\widehat{D}| e^{\chi_{2}}\left(1+\frac{|\widehat{C}|}{|\widehat{D}|} e^{-2 \chi_{2}}\right) \quad \text { if } 0 \leq \theta \leq \pi-\delta, \\
|\widehat{C}| e^{-\chi_{2}}\left(1+\frac{|\widehat{D}|}{|\widehat{C}|} e^{2 \chi_{2}}\right) \quad \text { if }-\pi+\delta \leq \theta<0
\end{array}\right. \\
& \leq \frac{\widehat{\Delta}_{1}}{r} e^{\left|\chi_{2}\right|}, \quad \text { if } 0 \leq|\theta| \leq \pi-\delta .
\end{aligned}
$$

On the other hand, we have for all sufficiently large $r$,

$$
\begin{aligned}
& \left|C e^{i \chi(L, \eta ; \zeta)}+D e^{-i \chi(L, \eta ; \zeta)}\right|=\left|\widehat{C} e^{i\left(\chi_{1}+i \chi_{2}\right)}+\widehat{D} e^{-i\left(\chi_{1}+i \chi_{2}\right)}\right| \\
& = \begin{cases}\left|\widehat{D} e^{-i \chi_{1}} e^{\chi_{2}}\left(1+\frac{\widehat{C}}{\widehat{D}} \cdot e^{2 i \chi_{1}} e^{-2 \chi_{2}}\right)\right| & \text { if } 0 \leq \theta \leq \pi-\delta, \\
\left|\widehat{C} e^{i \chi_{1}} e^{-\chi_{2}}\left(1+\frac{\widehat{D}}{\widehat{C}} \cdot e^{-2 i \chi_{1}} e^{2 \chi_{2}}\right)\right| & \text { if }-\pi+\delta \leq \theta<0 .\end{cases}
\end{aligned}
$$

We now choose

$$
r_{p}=\pi(p-\sqrt{p})-\frac{1}{2} \arg \left(\frac{\widehat{C}}{\widehat{D}}\right),
$$

where the principal value of the argument is chosen and $p$ is a suitably chosen large integer. Since the parameter $\eta=\eta_{1}+i \eta$ and the argument of $\zeta=r e^{i \theta}$ are fixed, so we may chose the integer $p$ so large so that $r=|\zeta|=r_{p}$ satisfies

$$
\left|-\eta_{1} \log r_{p}+\eta_{2} \theta\right| \leq \text { (const. ) } \sqrt{p} \text {. }
$$


We further assume that

$$
0 \leq|\theta|<\left(\frac{\pi}{4 r_{p}}\right)^{\frac{1}{2}}
$$

Then as in the proof of Lemma 2.1 we have

$$
2 r_{p}-\frac{\pi}{4}<2 r_{p}\left(1-\frac{\theta^{2}}{2}\right)<2 r_{p} \cos \theta<2 r_{p}
$$

We deduce from (6.7) and (6.12) that

$$
2 p \pi(1+o(1))-\frac{\pi}{4}<\arg \left(\frac{\widehat{C}}{\widehat{D}} e^{2 i \chi_{1}} e^{-2 \chi_{2}}\right)<2 p \pi(1+o(1)) .
$$

We deduce that if (6.13) holds then

$$
\Re\left(\frac{\widehat{C}}{\widehat{D}} e^{2 i \chi_{1}} e^{-2 \chi_{2}}\right)>0, \text { and } \Re\left(\frac{\widehat{D}}{\widehat{C}} e^{-2 i \chi_{1}} e^{2 \chi_{2}}\right)>0 .
$$

Thus we obtain from $(6.8),(6.11),(6.14)$ that if (6.13) holds, then

$$
\begin{aligned}
\mid C e^{i \chi(L, \eta ; \zeta)}+D e^{-i \chi(L, \eta ; \zeta) \mid} & =\left|\widehat{C} e^{i\left(\chi_{1}+i \chi_{2}\right)}+\widehat{D} e^{-i\left(\chi_{1}+i \chi_{2}\right)}\right| \\
& \geq \begin{cases}|\widehat{D}| e^{\chi_{2}} & \text { if } 0 \leq \theta<\left(\frac{\pi}{4 r_{p}}\right)^{\frac{1}{2}} \\
|\widehat{C}| e^{-\chi_{2}} & \text { if }-\left(\frac{\pi}{4 r_{p}}\right)^{\frac{1}{2}}<\theta<0\end{cases} \\
& = \begin{cases}|\widehat{D}| \max \left\{e^{\chi_{2}}, e^{-\chi_{2}}\right\}, \quad \text { if } 0 \leq \theta<\left(\frac{\pi}{4 r_{p}}\right)^{\frac{1}{2}}, \\
|\widehat{C}| \max \left\{e^{-\chi_{2}}, e^{\chi_{2}}\right\}, \quad \text { if }-\left(\frac{\pi}{4 r_{p}}\right)^{\frac{1}{2}}<\theta<0\end{cases} \\
& \geq \widehat{\Delta}_{2} \max \left\{e^{\chi_{2}}, e^{-\chi_{2}}\right\}, \quad \text { if } 0 \leq|\theta|<\left(\frac{\pi}{4 r_{p}}\right)^{\frac{1}{2}} \\
& =\widehat{\Delta}_{2} e^{\left|\chi_{2}\right|},
\end{aligned}
$$

where $\widehat{\Delta}_{2}=\min \{\widehat{C}, \widehat{D}\}$.

It remains to consider the case when $(6.13)$ is false. That is, we assume

$$
\left(\frac{\pi}{4 r_{p}}\right)^{\frac{1}{2}} \leq|\theta| \leq \pi-\delta
$$

Without loss of generality, we may assume that the integer $p$ is chosen large enough so that

$$
\left(\frac{\pi}{4 r_{p}}\right)^{\frac{1}{2}}<\delta
$$


Then (6.16) yields

$$
\left|2 r_{p} \sin \theta\right|>2 r_{p} \sin \left(\frac{\pi}{4 r_{p}}\right)^{\frac{1}{2}}>2 r_{p} \frac{2}{\pi}\left(\frac{\pi}{4 r_{p}}\right)^{\frac{1}{2}}=\left(\frac{4 r_{p}}{\pi}\right)^{\frac{1}{2}},
$$

and hence

$$
\left|2 \chi_{2}\right|=\left|2\left(r_{p} \sin \theta-\eta_{2} \log r-\eta_{1} \theta\right)\right|=\left(\frac{4 r_{p}}{\pi}\right)^{\frac{1}{2}}(1+o(1)) .
$$

We deduce from (6.11) that

(6.17)

$$
\begin{aligned}
& \left|C e^{i \chi(L, \eta ; \zeta)}+D e^{-i \chi(L, \eta ; \zeta)}\right|=\left|\widehat{C} e^{i\left(\chi_{1}+i \chi_{2}\right)}+\widehat{D} e^{-i\left(\chi_{1}+i \chi_{2}\right)}\right| \\
& \geq \begin{cases}\left|\widehat{D} e^{-i \chi_{1}} e^{\chi_{2}}\left(1-\left|\frac{\widehat{C}}{\widehat{D}}\right| \cdot e^{-\left(4 r_{p} / \pi\right)^{\frac{1}{2}}(1+o(1))}\right)\right| & \text { if }\left(\frac{\pi}{4 r_{p}}\right)^{\frac{1}{2}} \leq \theta \leq \pi-\delta, \\
\mid \widehat{C} e^{i \chi_{1}} e^{-\chi_{2}}\left(1-\left|\frac{\widehat{D}}{\widehat{C}}\right| \cdot e^{\left.-\left(4 r_{p} / \pi\right)^{\frac{1}{2}(1+o(1))}\right) \mid} \quad \text { if }-\pi+\delta \leq \theta \leq-\left(\frac{\pi}{4 r_{p}}\right)^{\frac{1}{2}}\right.\end{cases} \\
& \geq \frac{\widehat{\Delta}_{2}}{2} e^{\left|\chi_{2}\right|},
\end{aligned}
$$

whenever (6.16) holds and the integer $p$ is chosen sufficiently large.

We deduce from (6.11) and (6.15) that (6.17) now holds for $|\arg \zeta| \leq \pi-\delta$. We finally deduce from (6.10) and (6.17) that the inequality (6.6) holds on the boundary of the semi-annular region $\Omega\left(r_{p}, r_{q} ; \delta\right)$ where $q>p$. Rouché's theorem implies that the function (6.4) has the same number of zeros as the function

$$
C e^{i \chi(L, \eta ; \zeta)}+D e^{-i \chi(L, \eta ; \zeta)}
$$

in $\Omega\left(r_{p}, r_{q} ; \delta\right)$ for $r_{p}$ large. Clearly the above argument is valid for each $\delta>0$.

The zeros of (6.18) in the $\chi$-plane are given by

$$
\chi=\left[\frac{-1}{2 i} \log \left(\left(-\frac{C}{D}\right)+2 \pi i k\right)\right],
$$

for all integers $k$ sufficiently large. But

$$
\chi=\chi(L, \eta ; \zeta)=\zeta(1+o(1)),
$$

as $\zeta \rightarrow \infty$ in $\Omega(r, R ; \delta)$. Thus, the zeros (6.19) in the complex $\chi$-plane are mapped onto the $\Omega\left(r_{p}, r_{q} ; \delta\right)$ by $\chi$ with only a small perturbation. The images of the zeros in $\Omega\left(r_{p}, r_{q} ; \delta\right)$ tends to the locations given by $(6.19)$ as their moduli tend to infinity. We therefore conclude that $(6.18)$ has

$$
\frac{2 r_{q}}{\pi}+O(1)
$$

many zeros as $q \rightarrow+\infty$ for a fixed $p$. In fact, the zeros in (6.19) have exponent of convergence equal to one. In particular, (2.7) and (2.8) hold. 
Remark 6.2 Since we have only used the first leading terms in the asymptotic expansions (A.7) and (A.8) in the proof above, so it is not difficult to verify that the conclusion of the lemma still holds if we replace the assumption (6.4) by the asymptotic relation:

$$
V(\zeta) \sim C \psi_{+}(L, \eta ; \zeta)+D \psi_{-}(L, \eta ; \zeta),
$$

or even with the leading asymptotic terms only:

$$
V(\zeta) \sim C e^{i \chi(L, \eta ; \zeta)}+D e^{-i \chi(L, \eta ; \zeta)} .
$$

Remark 6.3 Although the argument used in the above proof is valid in the region in $-\pi<\arg \zeta<\pi$ by the asymptotic expansions (A.7) and (A.8) of the modified Coulomb wave functions, the corresponding asymptotic formulae exist when the phase angle differs from the above range by any integral multiple of $2 \pi i$ (see (A.11) and $\left.\left[17,\left(8.8 \mathrm{a}^{\prime}\right)\right]\right)$.

Next we state a lemma whose proof is similar to the proof of Lemma 2.2.

Lemma 6.4 Let $\alpha, C$ and $D$ be three non-zero constants, and let $L$ and $\eta$ be arbitrary constants. Then the entire function

$$
C \psi_{+}\left(L, \eta ; \alpha e^{-z}\right)+D \psi_{-}\left(L, \eta ; \alpha e^{-z}\right)
$$

has an infinite exponent of convergence of zeros.

Remark 6.5 In view of Remark 6.2, the conclusion of the above lemma clearly holds under the weaker assumption that

$$
W\left(\alpha e^{-z}\right) \sim C \psi_{+}\left(L, \eta ; \alpha e^{-z}\right)+D \psi_{-}\left(L, \eta ; \alpha e^{-z}\right),
$$

or even with only

$$
W\left(\alpha e^{-z}\right) \sim C e^{i \chi\left(L, \eta ; \alpha e^{-z}\right)}+D e^{-i \chi\left(L, \eta ; \alpha e^{-z}\right)} .
$$

\section{Lommel-Transform on Coulomb wave Equation}

We apply the Lommel-transformation (3.1) to the Coulomb wave equation (1.20) and obtain

$$
\begin{aligned}
t^{2} u^{\prime \prime}(t)+(1+ & 2 \gamma-\beta) t u^{\prime}(t) \\
& +\left[\alpha^{2} \beta^{2} t^{2 \beta}-2 \eta \alpha \beta^{2} t^{\beta}+\gamma(\gamma-\beta)-L(L+1) \beta^{2}\right] u(t)=0 .
\end{aligned}
$$

The solution of the transformed equation (7.1) is now given by

$$
\begin{aligned}
u(t)=t^{-\gamma} y(x) & =t^{-\gamma} y\left(\alpha t^{\beta}\right) \\
& =t^{-\gamma}\left[A F_{L}\left(\eta, \alpha t^{\beta}\right)+B G_{L}\left(\eta, \alpha t^{\beta}\right)\right] .
\end{aligned}
$$


We now make a further change of variable of the type (3.4) that transforms (7.1) to

$$
\begin{aligned}
f^{\prime \prime}(z)+m(2 \gamma-\beta) f^{\prime}(z) & +m^{2}\left[\alpha^{2} \beta^{2} e^{2 \beta m z}\right. \\
& \left.-2 \eta \alpha \beta^{2} e^{\beta m z}+\gamma(\gamma-\beta)-L(L+1) \beta^{2}\right] f(z)=0 .
\end{aligned}
$$

The solution to the above equation is then given by

$$
\begin{aligned}
f(z)=u(t) & =u\left(e^{m z}\right) \\
& =e^{-\gamma m z}\left[A F_{L}\left(\eta, \alpha e^{m \beta z}\right)+B G_{L}\left(\eta, \alpha e^{m \beta z}\right)\right] .
\end{aligned}
$$

\section{Proofs of Theorems 1.3 and 1.6}

Proof of Theorem 1.3 Let us choose $K_{-2}=-1 / 4, K_{-1}=1 / 2$ in (1.25) in Theorem 1.6. Then (1.27) gives

$$
\alpha_{ \pm}= \pm \frac{i}{2} \quad \text { and } \quad \eta_{ \pm}=\frac{1}{-4 \alpha_{ \pm}}= \pm \frac{i}{2} .
$$

We also have

$$
\widehat{n}=\frac{i K_{-1}}{\sqrt{K_{-2}}}=1 .
$$

We deduce (1.22) from (1.29) that

$$
K=-\left(L+\frac{1}{2}\right)^{2}=-\left(n_{ \pm}+\frac{1}{2} \pm \frac{1}{2}\right)^{2}=\left\{\begin{array}{c}
-((n-1)+1 / 2+1 / 2)^{2} \\
-(n+1 / 2-1 / 2)^{2}
\end{array}\right\}=-n^{2}
$$

We further deduce that

$$
a_{ \pm}=2\left(1+i \eta_{ \pm}\right)=2(1 \mp 1 / 2)=\left\{\begin{array}{l}
1 \\
3
\end{array}\right.
$$

and

$$
b_{ \pm}=-2 i \alpha_{ \pm}=\left\{\begin{array}{l}
1 \\
-1 .
\end{array}\right.
$$

Substituting all the above constants into Theorem 1.6 completes the proof.

Proof of Theorem 1.6 Let $f(z)$ be a non-trivial solution of (1.25). Then we get (7.3) by applying the Lommel transformation (3.1) to the Coulomb wave equation (1.20). Comparing the coefficients in equations (1.25) and (7.3), we deduce that $m=-1$, $\beta=1, \gamma=\beta / 2=1 / 2$,

$$
\alpha^{2}=K_{-2}, \quad-2 \eta \alpha=K_{-1}, \quad-1 / 4-L(L+1)=K_{0} .
$$


This and (7.4) give (1.27), (1.28), and that two linearly independent solutions to (1.25) are given by (1.26) as stated in the Theorem. We may assume, in addition, that the coefficient $K_{-1} \neq 0$ since otherwise the equation can be transformed to equation (1.10).

Suppose now that $\lambda(f)<+\infty$. Let $x=e^{-z}$. Then

$$
f(z)=V(x)=A G_{L}(\eta, \alpha x)+B F_{L}(\eta, \alpha x) .
$$

As in the proof of Theorem 1.2, we rewrite (8.2) in terms of the modified Coulomb wave functions $\psi_{+}$and $\psi_{-}$(defined in (6.3)) in the form of

$$
V(x)=C \psi_{+}(L, \eta ; \alpha x)+D \psi_{-}(L, \eta ; \alpha x)
$$

where the constants $C$ and $D$ are as given in (5.3). We observe from the definitions of the modified Coulomb wave functions that $V(x)$ given by $(8.3)$ is analytic in $\mathcal{C}$. It follows from Lemmas 6.1 and 6.4 that if $\lambda(f)<+\infty$ for a non-trivial solution, then exactly one of the constants $C$ and $D$ must be zero. Without loss of generality, we may assume that $C=1, D=0$, i.e., $A=-i B$. It remains to investigate when

$$
\lambda\left(\psi_{+}\left(L, \eta, \alpha e^{-z}\right)\right)<+\infty .
$$

But (8.4) must hold for each branch, including the principal branch of $\psi_{+}$. However, we have

$$
f(z)=\psi_{+}\left(L, \eta, \alpha e^{-z}\right)=\psi_{+}\left(L, \eta, \alpha e^{-z} e^{2 i m \pi}\right)
$$

where the last term above is given by an analytic continuation of the $\psi_{+}$. Since the asymptotic expansion of a branch of $\psi_{+}$in the phase range $(2 m-2) \pi+\varepsilon<\arg \rho<$ $2 m \pi-\varepsilon(m \in \mathbb{Z})^{5}$ is given by (A.11) [17, (8.8a), p. 6164], ${ }^{6}$ so we have

$$
\psi_{+}(L, \eta ; \alpha \zeta) \sim C e^{i \chi(L, \eta ; \alpha \zeta)}+D e^{-i \chi(L, \eta ; \alpha \zeta)},
$$

where

$$
C=\frac{e^{-2(m-1) \pi \eta}}{\sin (2 \pi L)}\left\{\sin (2 m \pi L)-e^{-2 \pi \eta} \sin [2(m-1) \pi L]\right\}
$$

and

$$
D=-\frac{e^{+2 m \pi \eta}}{\sin (2 \pi L)} \sin (2 m \pi L)\left\{1-e^{-2 \pi i(L-i \eta)}\right\}
$$

According to Remark 6.5, in order for the asymptotic relation (6.22) for any nontrivial solution to satisfy (1.3), exactly one of the coefficients of (6.22) must be zero.

\footnotetext{
${ }^{5}$ We have used the same notation to denote different branches of $\psi_{+}$that differ from the principal branch by an integral multiple of $2 \pi$.

${ }^{6}$ We note that the authors in [17, p. 6163] applied (A.10) repeatedly to obtain an analytic continuation formula for $\psi_{+}$for general phase angles. They then use this formula together with (A.7), (A.8) to obtain the asymptotic expansion (A.10) as stated in the appendix. However, the authors did not explicitly give the analytic continuation formula for general phase angles in [17]. But formula (A.11) suffices for our application here.
} 
In other words, exactly one of (8.5) or (8.6) above must be zero for each non-zero integer $m$. Since we have assumed that $K_{-1} \neq 0$, equation (8.1) implies that $\eta \neq 0$ as well.

Suppose we have

$$
L=k, k \in \mathbb{Z} \quad \text { or } \quad L=k+\frac{1}{2}, k \in \mathbb{Z}
$$

Then a simple application of L'Hospital's rule to (8.5) as $L \rightarrow k$ or $L \rightarrow k+1 / 2$, for each integer $k$, shows that

$$
e^{-2 \pi \eta}=\frac{m}{m-1}
$$

which never holds. Hence $c \neq 0$ when (8.7) holds. If we now apply L'Hospital's rule to (8.6) as $L \rightarrow k$ or $L \rightarrow k+1 / 2$, for each integer $k$, then we obtain

$$
D=-e^{+2 m \pi \eta} m\left\{1-e^{-2 \pi i(L-i \eta)}\right\} .
$$

which is zero if and only if $1-e^{-2 \pi i(L-i \eta)}=0$. Combining (8.8), (8.9) with Lemmas 6.1 and 6.4 , we obtain a contradiction to $(8.4)$ unless $1-e^{-2 \pi i(L-i \eta)}=0$. That is,

$$
L-i \eta=n
$$

for some integer $n$. We deduce from (A.5) that $n$ must be non-negative.

We assume henceforth that

$$
L \neq k, k \in \mathbb{Z} \quad \text { and } \quad L \neq k+\frac{1}{2}, k \in \mathbb{Z} \text {. }
$$

We first assume that $C$ is zero, then (8.5) implies

$$
\sin (2 m \pi L)=e^{-2 \pi \eta} \sin [2(m-1) \pi L]
$$

which can be written in the form

$$
e^{-2 \pi i(L-i \eta)}=\frac{1-e^{-4 m \pi L i}}{1-e^{-4(m-1) \pi L i}}
$$

If we denote $S=e^{-2 \pi i(L-i \eta)}$, then the above equation becomes

$$
1-e^{-4 m \pi L i}=S\left(1-e^{-4(m-1) \pi L i}\right)
$$

for all non-zero integer $m$. We now distinguish three cases.

(i) If $\Im(L)<0$, then both $1-e^{-4 m \pi L i}$ and $1-e^{-4(m-1) \pi L i}$ tend to 1 as $m \rightarrow+\infty$. We deduce $S=1$. But then (8.14) yields $e^{4 \pi L i}=1$, i.e., $L=k / 2, k \in \mathbb{Z}$. This contradicts (8.11) and the assumption that $\Im(L)>0$. 
(ii) If $\Im(L)>0$, then since equation (8.12) is independent of the sign of $L$, we may replace $L$ by $-L$. Thus, we may appeal to part (i) and get a contradiction as in (i).

(iii) We now assume that $\Im(L)=0$. Hence $L$ is real and

$$
\left|e^{-4 m \pi L i}\right|=1 \quad \text { and } \quad\left|e^{-4(m-1) \pi L i}\right|=1 .
$$

If we iterate equation (8.14) $m-2$ times, then we have

$$
1-e^{-4 m \pi L i}=S^{m-1}\left(1-e^{-4 \pi L i}\right) .
$$

If we let $m \rightarrow+\infty$, then either $|S|<1$ or $|S|>1$ will lead to a contradiction, unless $L$ is an integer. This also contradicts (8.11). We thus assume that $|S|=1$. Since $L$ is real, so $|S|=1$ implies that $\eta=i l$ for some integer $l$. Hence

$$
\pm 1=s=e^{-2 \pi i L}
$$

showing that $L$ must also be either an integer or half an integer. This again contradicts (8.11).

This shows that the constant $C$ given by (8.5) cannot be zero. Thus, we must have $D$ in (8.6) to be zero. We deduce $1-e^{-2 \pi i(L-i \eta)}=0$, and this implies that

$$
L-i \eta=n
$$

for some integer $n$. We notice from (A.5) that $n$ must be non-negative.

By the analytic continuation principle, the above argument is independent of any particular branch chosen except for the principal branch. The identity theorem implies that there must be a unique non-negative integer $n$ amongst all branches except perhaps for the principal branch, but otherwise arbitrary, for which (8.10) and (8.15) hold. It remains to consider the principal branch of (1.32). If (1.32) has a finite number of zeros in $\mathcal{C}$, then the identity theorem implies that the value of $L-i \eta$ must be amongst the non-negative integers $n$ found earlier. But it is known that when (8.15) holds, then (1.32) has a finite number of zeros for each non-negative integer $n$. This conclusion follows from the properties (A.12) and (A.13). This proves that for each branch of $-\pi+2 \pi k<\arg \zeta<\pi+2 \pi k(k=0, \pm 1, \pm 2, \ldots)$, if (1.32) has a finite number of zeros there, then (8.15) holds. We deduce from (8.2) that $V(x)=C \psi_{+}(L, \eta ; \alpha x)$.

An inspection of the two subscripts of the Whittaker functions in (A.12) implies that the generalized Bessel polynomial can be written in terms of the Whittaker functions from (A.13) by choosing $a=2(L-n)=2(1+i \eta)$. That is, $L=a / 2+n$ and $-i \eta=1-a / 2$. In particular, it shows, as expected, that the principal branch of (A.12) has only finitely many zeros when $L-i \eta=n$.

It follows from (8.1) that there are two values of $\alpha$ and hence two values of $\eta$ such that

$$
\alpha=\alpha_{ \pm}= \pm \sqrt{K_{-2}}, \quad-i \eta=-i \eta_{ \pm}= \pm \frac{i K_{-1}}{2 \sqrt{K_{-2}}} .
$$


Thus

$$
a_{ \pm}:=2\left(1+i \eta_{ \pm}\right)=2 \mp \frac{i K_{-1}}{\sqrt{K_{-2}}} .
$$

We deduce from (8.15) and (8.16) that

$$
L-i \eta_{+}=n_{+}, \quad L-i \eta_{-}=n_{-},
$$

where both $n_{+}$and $n_{-}$are non-negative integers, and

$$
\frac{i K_{-1}}{\sqrt{K_{-2}}}=\left(-i \eta_{+}\right)-\left(-i \eta_{-}\right)=i\left(\eta_{-}-\eta_{+}\right)=n_{+}-n_{-} .
$$

We may assume that $n_{+}>n_{-} \geq 0$ because of the assumption that $K_{-1} \neq 0$.

In (8.1), we set

$$
L+\frac{1}{2}=i \sqrt{K_{0}},
$$

and thus (8.18) together with (8.16) and (8.19) give

$$
i \sqrt{K_{0}}=L+\frac{1}{2}=\frac{1}{2}+i \eta_{ \pm}+n_{ \pm}=\frac{1}{2} \mp \frac{i K_{-1}}{2 \sqrt{K_{-2}}}+n_{ \pm} .
$$

This proves (1.28) and (1.29).

Substituting (8.19) into (8.21) gives (1.28). It is easy to deduce (1.29) from (1.28). This proves that (1.28) and (1.29) are equivalent.

Let $b_{ \pm}=-2 i \alpha_{ \pm}$. We see from (8.2), $A=-i B,(6.3),($ A.12), (A.13) and (8.17) that

$$
\begin{aligned}
f_{+}(z) & =e^{z / 2}\left[A G_{L}\left(\eta_{+}, \alpha_{+} e^{-z}\right)+B F_{L}\left(\eta_{+}, \alpha_{+} e^{-z}\right)\right] \\
& =-i B e^{z / 2}\left[G_{L}\left(\eta_{+}, \alpha_{+} e^{-z}\right)+i F_{L}\left(\eta_{+}, \alpha_{+} e^{-z}\right)\right] \\
& =-i B \cdot e^{z / 2} \psi_{+}\left(L, \eta_{+}, \alpha_{+} e^{-z}\right) \\
& =\widehat{B} \cdot e^{z / 2} W_{-i \eta_{+}, L+1 / 2}\left(-2 \alpha_{+} e^{-z}\right) \\
& =\widehat{B} \cdot e^{z / 2} W_{1-\frac{a_{+}}{2}, \frac{a_{+}-1}{2}+n_{+}}\left(-2 i \alpha_{+} e^{-z} \cdot e^{-i \pi / 2}\right) \\
& =\widehat{B} \cdot e^{z / 2} W_{1-\frac{a_{+}}{2}, \frac{a_{+}-1}{2}+n_{+}}\left(b_{+} e^{-z}\right) \\
& =\widehat{B} \cdot e^{z / 2} y_{n_{+}}\left(e^{z} ; a_{+}, b_{+}\right)\left(b_{+} e^{-z}\right)^{1-a_{+} / 2} \exp \left(-b_{+} e^{-z} / 2\right) \\
& =\widehat{B} \cdot e^{z / 2} e^{-\left(1-a_{+} / 2\right) z} y_{n_{+}}\left(e^{z} ; a_{+}, b_{+}\right) \exp \left(-b_{+} e^{-z} / 2\right) .
\end{aligned}
$$

We only need to replace all the subscripts + by - in the above derivation in order to obtain a second linearly independent solution $f_{-}(z)$ as stated in the theorem.

Conversely, suppose we are given non-negative integers $n_{+}$and $n_{-}, n_{+}>n_{-} \geq 0$ such that (1.28) or (1.29) is satisfied. Define $a_{ \pm}=2\left(1+i \eta_{ \pm}\right)$and $b_{ \pm}=-2 i \alpha_{ \pm}$such that (1.27) holds, then the functions (1.30) and (1.31) are two linearly independent solutions of (1.25) each with finite exponent of convergence of zeros. This completes the proof of the theorem. 


\section{The Remaining Case $\ell \geq 3$ and a Problem of Heine}

We have already dealt with the equation (1.1) when $\ell=1,2$. We now consider the equation when $\ell \geq 3$. In view of Remark 1.9, it suffices to consider the case where $\ell \geq 3$ and $k=0$.

As already mentioned in the introduction, there is no suitable special function theory for function as solutions to (3.2) when $n^{\prime}=0$ and $n>2$ other than the Bessel and Coulomb wave functions $\left(n^{\prime}=0\right.$ and $\left.n=2\right)$. This is discussed in more detail in $\S 10.4$.

We shall, as in the case of Bank [4], suppose that (1.1) has a solution $f(z)$ with $\lambda(f)<+\infty$. Hence $f(z)$ must admit either the representation (1.4) or (1.5) depending on whether $\ell$ is odd or even, respectively [14, Prop. 1]. However, instead of constructing an explicit solution as in [4], we relate the problem to a problem of Heine and give an entirely new combinatorial-type result.

Let us write $f(z)$ in the form

$$
f(z)=\psi\left(e^{z / q}\right) \exp \left(P\left(e^{z / q}\right)+z d\right),
$$

where

$$
P(\zeta)=\sum_{j=1}^{\ell_{i}} d_{j} \zeta^{j}, \quad i=1,2
$$

and where $q=1, \ell_{1}=\ell / 2$ if $\ell$ is even and $q=2, \ell_{2}=\ell$ when $\ell$ is odd [14, Prop. 1], $d$ is a constant and $\psi(\zeta)$ is a polynomial of the form:

$$
\psi(\zeta)=c_{n} \zeta^{n}+\cdots+c_{0} .
$$

We may assume that $P(0)=0$ in (9.2). Also by adjusting the value of $d$ in (9.1), we may assume $c_{0} \neq 0$ in (9.3). It is a routine calculation to show that the $\psi(\zeta)$ in (9.1) satisfies the following differential equation

$$
\begin{aligned}
\zeta^{2} \psi^{\prime \prime}(\zeta)+\left(2 \zeta^{2} P^{\prime}(\zeta)\right. & +(2 d q+1) \zeta) \psi^{\prime}(\zeta)+\left\{\zeta^{2} P^{\prime \prime}(\zeta)+\left(\zeta P^{\prime}(\zeta)\right)^{2}\right. \\
& \left.+(2 d q+1) \zeta P^{\prime}(\zeta)+q^{2}\left(d^{2}+\sum_{j=0}^{\ell_{i}} K_{j} \zeta^{q j}\right)\right\} \psi(\zeta)=0
\end{aligned}
$$

In the case when $\ell$ is odd, we shall give a general condition that relates the polynomial $P(\zeta)$ and the coefficients $K_{j}, j=0, \ldots, \ell$ that must be satisfied in order for (1.1) (with $\ell>0$ and $k=0$ in (1.2)) to admit a solution $f$ that satisfies (1.3).

We first recall the following result due to one of the authors [14]:

Theorem $D$ Let $\ell$ and $k$ be integers such that $\ell$ is odd and $\ell \geq k=0$. Suppose that the equation (1.1) with the coefficient $A(z)$ given by (1.2) admits a zero-free solution $f(z)$, then $K_{j}=0, j=1, \ldots, \ell-1, K_{0} \neq 0$. Moreover,

$$
K_{0}=-\frac{\ell^{2}}{16}
$$




$$
f(z)=\exp \left(d_{\ell} e^{\ell z / 2}-\ell z / 4\right)
$$

and $\ell^{2} d_{\ell}^{2}+4 K_{\ell}=0$.

The following theorem allows us to consider solutions $f(z)$ to (1.1) with $0 \leq$ $\lambda(f)<+\infty$.

Theorem 9.1 Assume that $\ell, \ell \geq 3$ is odd and that the coefficients $K_{\ell}, \ldots, K_{(\ell+1) / 2}$ are given in (1.2). Then there are at most

$$
2\left(\begin{array}{c}
n+(\ell-1) / 2 \\
n
\end{array}\right)
$$

choices of the remaining coefficients $K_{(\ell-1) / 2}, \ldots, K_{1}$, such that for each of the choices the differential equation (1.1) admits a solution given by (9.1) with the polynomial $\psi(\zeta)$ of degree $n$ given by (9.3).

The proof of this theorem is based on the following problem studied by Heine.

Theorem $E$ (Heine [26, pp. 472-479]) Let $\Pi_{\ell+2}(\zeta)$ and $\Pi_{\ell+1}(\zeta)$, be given polynomials of degrees $\ell+2$ and $\ell+1$, respectively. Then there are at most

$$
\left(\begin{array}{c}
n+\ell \\
n
\end{array}\right)
$$

determinations of polynomials $\Pi_{\ell}(\zeta)$ of degree $\ell$ such that for each of these polynomials the differential equation

$$
\Pi_{\ell+2}(\zeta) y^{\prime \prime}(\zeta)+\Pi_{\ell+1}(\zeta) y^{\prime}(\zeta)+\Pi_{\ell}(\zeta) y(\zeta)=0
$$

admits a polynomial solution $y(\zeta)$ of exact degree $n$.

Remark 9.2 In general the number of choices of $\Pi_{\ell}(\zeta)$ in the Theorem E is exactly $\left(\begin{array}{c}n+\ell \\ n\end{array}\right)$ (see Szego" [38, p. 151]). This implies the choices of the remaining coefficients $K_{(\ell-1) / 2}, \ldots, K_{1}$ asserted in Theorem 9.1 have the lower bound

$$
\left(\begin{array}{c}
n+(\ell-1) / 2 \\
n
\end{array}\right)
$$

However, we do not know under what condition that the number of choices of the coefficients $\Pi_{\ell}(\zeta)$ matches the upper bound $\left(\begin{array}{c}n+\ell \\ n\end{array}\right)$, except for the special case as described in Szegő [38, pp. 151-155].

Remark 9.3 Suppose the $k, 0 \leq k \leq \ell-1$, coefficients of the polynomial $\Pi_{\ell}(\zeta)$ of (9.7) are fixed, then according to the argument used by Heine [26], the upper bound of the number of choices (9.6) of the $\Pi_{\ell}$ and the polynomial solutions $\psi$ of (9.7) is reduced to

$$
\left(\begin{array}{c}
n+\ell-k \\
n
\end{array}\right)
$$


Remark 9.4 We note that Heine's result is an existence theorem, that is, it does not give a constructive way to find the polynomial $\Pi_{\ell}(\zeta)$ or the corresponding polynomial solution $y(\zeta)$ asserted in Theorem E. However, Heine's theorem is a fundamental result in electrostatic models of orthogonal polynomials [38], and in quantized physical models $[29,30]$.

Proof of Theorem 9.1 Suppose that (1.1) admits a solution $f(z)$ that satisfies (1.3), hence Theorem A implies that $f(z)$ has a representation in the form of (9.1). Thus $f(z)$ is given by (9.1) with $q=2$ and $P(\zeta)$ in (9.4) has degree $\ell$.

Substituting $f(z)$ into (1.1) gives the equation (9.4) satisfied by the polynomial (9.3) with $P(\zeta)$ given by (9.2). We may assume without loss of generality that the coefficient $c_{n}=1$ in (9.3).

We now note that the equation (9.4) can be written in the form

$$
\Pi_{2}(\zeta) y^{\prime \prime}(\zeta)+\Pi_{\ell+1}(\zeta) y^{\prime}(\zeta)+\Pi_{\ell}(\zeta) y(\zeta)=0,
$$

where

$$
\Pi_{2}(\zeta)=\zeta^{2}, \quad \Pi_{\ell+1}(\zeta)=2 \zeta^{2} P^{\prime}(\zeta)+(4 d+1) \zeta
$$

and

$$
\Pi_{\ell}(\zeta)=\zeta^{2} P^{\prime \prime}(\zeta)+\left(\zeta P^{\prime}(\zeta)\right)^{2}+(4 d+1) \zeta P^{\prime}(\zeta)+4\left(d^{2}+\sum_{j=0}^{\ell} K_{j} \zeta^{2 j}\right) .
$$

Since the polynomial $\left(\zeta P^{\prime}(\zeta)\right)^{2}$ has degree $2 \ell$, the highest degree contribution comes from the term $\Pi_{\ell}(\zeta) \psi(\zeta)$ is $n+2 \ell$, whereas the highest degree contribution from the middle term $\Pi_{\ell+1}(\zeta) \psi^{\prime}(\zeta)$ of (9.9) is only $n+\ell$. Moreover, the highest degree from the term $\Pi_{2}(\zeta) y^{\prime \prime}(\zeta)$ in (9.9) is just $n$. It follows that the corresponding coefficients for the terms $\zeta^{\nu}, 1+\ell \leq \nu \leq 2 \ell$ in (9.11) must vanish. When $\nu=2 \ell$, the coefficient for $\zeta^{\nu}$ is

$$
\left(\ell^{2} d_{\ell}^{2}+4 K_{\ell}\right) c_{n}=0 .
$$

Since both $c_{n}$ and $K_{\ell}$ are assumed to be non-zero, so we deduce that $d_{\ell} \neq 0$. It will be sufficient to consider the coefficients of $\zeta^{\nu}$ in (9.11) for the remaining equations where $1+\ell \leq \nu \leq 2 \ell-1$ :

(A) $\nu$ is even and $1+\ell \leq \nu \leq 2 \ell-1$,

$$
\left(\frac{\nu}{2}\right)^{2} d_{\nu / 2}^{2}+\sum_{\substack{i+j=\nu \\ i<j}} 2 i j d_{i} d_{j}+4 K_{\nu / 2}=0 .
$$

(B) $\nu$ is odd and $1+\ell \leq \nu \leq 2 \ell-1$,

$$
\sum_{\substack{i+j=\nu \\ i<j}} 2 i j d_{i} d_{j}=0
$$


In case (B) above where $\nu$ is odd, a simple finite induction applied to (9.14) implies

$$
\text { all the } d_{j} \text {, with } j \text { even, must vanish, }
$$

as was already proved in [7] (see also [14, Prop. 1]). That is, all the equations in (9.14) vanish. We now analyze the equations described in (9.13). With a given value of $K_{\ell}$, the equation (9.12) can be solved to give two values of $d_{\ell}$, which we denote by $d_{\ell}$ and $-d_{\ell}$, say.

Let us take $\nu=2 \ell-2$ in (9.13). Then $\ell-1$ is even, so (9.15) implies that $d_{\ell-1}=0$. Hence

$$
2 \ell(\ell-2) d_{\ell} d_{\ell-2}+4 K_{\ell-1}=0
$$

Since $K_{\ell-1}$ is given and suppose we choose the value $d_{\ell}$, then the above equation will give a unique solution which we denote by $d_{\ell-2}$, say. However, if we choose the value $-d_{\ell}$ instead, then with the same given $K_{\ell-1}$, the equation (9.16) gives another unique solution which we denote by $-d_{\ell-2}$, say. Let us now consider $\nu=2 \ell-4$, then the equation (9.13) becomes

$$
\begin{aligned}
(\ell-2)^{2} d_{\ell-2}^{2}+2 \ell(\ell-4) & d_{\ell} d_{\ell-4}+2(\ell-1)(\ell-3) d_{\ell-1} d_{\ell-3}+4 K_{\ell-2} \\
& =(\ell-2)^{2} d_{\ell-2}^{2}+2 \ell(\ell-4) d_{\ell} d_{\ell-4}+4 K_{\ell-2}=0
\end{aligned}
$$

since (9.15) implies $d_{\ell-1} d_{\ell-3}=0$. It follows from (9.16), (9.17) and with the given $K_{\ell-2}$, that we can determine unique $d_{\ell-4}$ and $-d_{\ell-4}$ each for the two choices of $d_{\ell}$ and $-d_{\ell}$. We now apply finite induction to (9.13) to repeat the above calculation to conclude that the two sets of coefficients

$$
\left\{d_{\ell}, \ldots, d_{1}\right\}, \quad\left\{-d_{\ell}, \ldots,-d_{1}\right\}
$$

(solutions to equations (9.13)) obtained are uniquely determined by the coefficients $K_{\ell}, \ldots, K_{(\ell+1) / 2}$.

Let us now analyze the coefficients $A_{\nu}$ of $\zeta^{\nu}$ of $\Pi_{\ell}(\zeta)$ in (9.11) where $1 \leq \nu \leq \ell$. We again distinguish the cases of even and odd indices:

(C) $\nu$ is even and $1 \leq \nu \leq \ell$,

$$
A_{\nu}=\left(\frac{\nu}{2}\right)^{2} d_{\nu / 2}^{2}+\sum_{\substack{i+j=\nu \\ i<j}} 2 i j d_{i} d_{j}+4 K_{\nu / 2}
$$

(D) $\nu$ is odd and $1 \leq \nu \leq \ell$,

$$
A_{\nu}=\nu(4 d+\nu) d_{\nu}+\sum_{\substack{i+j=\nu \\ i<j}} 2 i j d_{i} d_{j}=\nu(4 d+\nu) d_{\nu}
$$

since (9.15) implies that all the $d_{j}=0$, for $j$ even. 
In particular, when $\nu=\ell+n$, then it follows from the higher powers of $\zeta$ in (9.19) when $\nu$ is even or from (9.20) when $\nu$ is odd, and the highest power of $\zeta$ in the term $\Pi_{\ell+1}(\zeta) \psi^{\prime}(\zeta)$ of equation (9.9) that the following equation

$$
2 n \ell d_{\ell}+\ell(4 d+\ell) d_{\ell}=0
$$

holds. That is,

$$
2 n+4 d+\ell=0
$$

since both $d_{\ell}$ and $\ell$ are non zero. This determines $d$ uniquely. The relation (9.21) was also proved in $[14,(2.17) \text { and Theorem } 4]^{7}$ by a different method.

It follows from (9.19), (9.20)) and (9.15) that each of the coefficients $K_{(\ell-1) / 2}, \ldots$, $K_{1}$ appears only in the coefficients of $\zeta^{\nu}$ in (9.4), $q=2$ when $\nu(\nu \leq \ell-1)$ is positive and even, and in a recursive manner as $\nu$ decreases from $\ell$ down to 1 . The remaining coefficients of $\zeta^{\nu}$ in (9.13) for $1 \leq \nu \leq \ell$, that is, those in (9.18) were already determined by the given $K_{j}, j=(\ell+1) / 2, \ldots, \ell$, as was shown in a previous argument.

Since both the coefficients $\Pi_{2}(\zeta)$ and $\Pi_{\ell+1}(\zeta)$ contain no constant term, and so the only constant term in $\Pi_{\ell}$ must satisfy

$$
d^{2}+K_{0}=0
$$

Recall that the value of $d$ is already determined by (9.21); we deduce that $K_{0}$ is also determined uniquely by (9.22). But since the value of $d$ depends only on $\ell$ and $n$, so is $K_{0}$. In particular, $K_{0}$ is a negative rational number.

We now apply the transformation

$$
\psi(\zeta)=\zeta^{n} \Psi(1 / \zeta)
$$

to equation (9.4) where $n$ is the degree of (9.3). Notice that $\Psi(t)$ is a polynomial of degree $n$ since we have assumed that $c_{0} \neq 0$. This yields an equation, after making the change of variable $t=1 / \zeta$ and multiplying the resulting equation by $t^{\ell-n}$ throughout,

$$
\widetilde{\Pi}_{\ell+2}(t) \Psi^{\prime \prime}(t)+\widetilde{\Pi}_{\ell+1}(t) \Psi^{\prime}(t)+\widetilde{\Pi}_{\ell}(t) \Psi(t)=0
$$

where

$$
\widetilde{\Pi}_{\ell+2}(t)=t^{\ell+2}, \quad \widetilde{\Pi}_{\ell+1}(t)=(-2 n+2) t^{\ell+1}-t^{\ell+2} \Pi_{\ell+1}(1 / t),
$$

and

$$
\widetilde{\Pi}_{\ell}(t)=n(n-1) t^{\ell}+t^{\ell} \Pi_{\ell}(1 / t)+n t^{\ell+1} \Pi_{\ell+1}(1 / t) .
$$

\footnotetext{
${ }^{7}$ Note that there is a misprint in $[14,(2.17)]$ : replace $\ell / 2$ by $\ell$.
} 
Clearly the degree of the coefficient $\widetilde{\Pi}_{\ell+2}(t)$ is $\ell+2$. We now claim that the coefficient $\widetilde{\Pi}_{\ell+1}(t)$ is a polynomial of degree $\ell+1$. This follows from $(9.2)\left(\ell_{i}=\ell_{2}=\ell\right),(9.10)$, and (9.25) that

$$
\widetilde{\Pi}_{\ell+1}(t)=(-2 n-4 d+1) t^{\ell+1}+\delta_{1} t^{\ell-1}+\cdots .
$$

Using (9.21) and noting that $\ell>0$ and $n \geq 1$, we deduce that the leading coefficient $-2 n-4 d+1=-\ell+1(\ell \geq 3)$ in (9.27) is never zero. This shows that $\widetilde{\Pi}_{\ell+1}$ has degree exactly $\ell+1$ and the claim is established.

We now show that $\widetilde{\Pi}_{\ell}(t)$ has degree equal to $\ell$. It is easy to verify from the definition of $\Pi_{\ell}(\zeta)$ in $(9.11)$ that

$$
n t^{\ell+1} \Pi_{\ell+1}(1 / t)=n(4 d+1) t^{\ell}+\cdots+2 n \ell d_{\ell} .
$$

Also, we have from (9.11) and from (9.20) when $\nu=\ell$ that

$$
t^{\ell} \Pi_{\ell}(1 / t)=\ell(4 d+\ell) d_{\ell}+\cdots+(\text { const. }) t^{\ell-1}=\delta_{2} t^{\ell-1}+\cdots .
$$

We deduce from the definition of $\widetilde{\Pi}_{\ell}(t)$ in (9.26) and the above two equations that

$$
\widetilde{\Pi}_{\ell}(t)=n(n+4 d) t^{\ell}+\delta_{3} t^{\ell-1}+\cdots .
$$

But (9.21) implies that $n(n+4 d)=(-n-\ell) n \neq 0$. We conclude that $\widetilde{\Pi}_{\ell}(t)$ has degree exactly $\ell$. Hence the degrees of the polynomial coefficients of the equation (9.24) satisfy the hypothesis of Theorem E.

So we obtain two sets of constants $\left\{d_{j}\right\}$ and $\left\{-d_{j}\right\}(1 \leq \nu \leq \ell)$ such that each of them is uniquely determined by the given constants $K_{j}, j=(\ell+1) / 2, \ldots, \ell$. Since the coefficients $\widetilde{\Pi}_{\ell+2}(\zeta)$ and $\widetilde{\Pi}_{\ell+1}(\zeta)$ of the equation (9.24) are given in terms of $\left\{d_{j}\right\}$ and $\left\{-d_{j}\right\}$, so we obtain two sets of equations (9.25) and (9.26) with $\left\{ \pm d_{j}\right\}$ as coefficients. The argument we use below is independent of the choice of the $\left\{ \pm d_{j}\right\}$ in (9.26). Thus, it suffices to consider the constants to be $\left\{d_{j}\right\}$. Then we easily deduce from (9.10), (9.11), (9.19), (9.20) and (9.26) that the coefficients $K_{j}$ only appear in (9.19) for $j=1, \ldots,(\ell-1) / 2$. Hence there are exactly $\frac{\ell-1}{2}$ of them. The remaining coefficients in (9.20) only depend on $\left\{d_{j}\right\}$ which were found earlier.

Hence the polynomial $\widetilde{\Pi}_{\ell}(t)$ has $\ell-(\ell-1) / 2=(\ell+1) / 2$ coefficients fixed and $(\ell-1) / 2$ of the $\left\{K_{j}\right\}$ are to be chosen. Remark 9.3 to Theorem E shows that there are at most

$$
\left(\begin{array}{c}
n+\ell-\frac{\ell+1}{2} \\
n
\end{array}\right)
$$

choices of $K_{j}, j=1,2, \ldots,(\ell-1) / 2$, such that for each of which (9.24) and hence (9.9) will admit a polynomial solution (9.2) of degree $n$. A similar argument applies for the set $\left\{-d_{j}\right\}$ and this again gives a set of $K_{j}, j=1,2, \ldots,(\ell-1) / 2$ with the same cardinality as in the previous case. This proves (9.5) as asserted in the theorem.

Remark 9.5 We are unable to find a similar result to Theorem 9.1 when $\ell$ is even. This is because of the corresponding formula analogues to (9.21) is very different (see [5, Lemma 3.1] or [14, (2.16)]), and that in general the consideration is more complicated when $\ell$ is even (cf. [14, Theorems 1-3]). 
Complex Oscillation Theory and Special Functions

\section{Concluding Remarks}

\subsection{Zero Distribution of Confluent Hypergeometric Functions}

The subsection focuses on some direct consequences of the main results (Theorems 1.2 and 1.6) in the zero distribution of the Bessel functions and Coulomb wave functions. In each of the two classes of functions that we shall consider below, it is known that when certain combinations of values of parameters are taken, the corresponding special functions have a finite number of zeros. These results usually appear in the form of a product which has a special polynomial component in its factorization. A main contribution from the proofs of Theorems 1.2 and 1.6 is to give converse statements of the above results, which appear to be new.

We first consider the Bessel and Hankel functions.

Theorem 10.1 Let $k$ be an integer, and let $A, B$ and $\nu$ be arbitrary complex constants. The expression (1.16) representing the branch defined in $\{\zeta:-\pi+k \pi<\arg \zeta<$ $\pi+k \pi\}$ admits a finite number of zeros if and only if $A=-i B$ and $\nu=n+\frac{1}{2}$, for some integer $n$. In particular, this implies that the Hankel function $H_{\nu}^{(1)}(\zeta)$ defined in $\{\zeta:-\pi+k \pi<\arg \zeta<\pi+k \pi\}$ admits a finite number of zeros if and only if $\nu=n+\frac{1}{2}$ for some integer $n$.

Proof If $A=-i B$ and $\nu=n+\frac{1}{2}$, for some integer $n$, then it is well known that (1.16) admits a finite number of zeros. In fact, the expression (1.16) reduces to $H_{n+1 / 2}^{(1)}(\zeta)$ which has only finitely many zeros [40, p. 298]. These confirm the first parts of the above (well known) statements. The converse parts are obtained by combining the proofs in $\S 2$ and $\S 5$ about the finiteness of (1.16) so that the corresponding entire function solution to (1.10) satisfies (1.3) (see Remark 5.2).

Knowledge of the zeros of the Bessel and Hankel functions has many physical applications [32, 19]. In [40, pp. 505-506], the zeros of $\cos \alpha J_{\nu}-\sin \alpha Y_{\nu}$ is mentioned, where $\alpha$ is a non-zero arbitrary complex number. However, there is no $\alpha$ that will satisfy $\cos \alpha=1$ and $\sin \alpha=-i$ which is exactly the case when the expression is reduced to have a Bessel polynomial component. So it does not cover our case. Numerical studies concerning the trajectories of the $z$-zeros of the Hankel functions as a function of its order $\nu$ are given in [15] for real $\nu$, and [37] for general $\nu$.

We now come to the Coulomb wave functions. We recall that in the proof of Theorem 1.6 in $\S 6$, using (A.12) and (A.13), the Hadamard factorization of the modified Coulomb wave function $\psi_{+}$has a polynomial component when $L-i \eta$ is a nonnegative integer. The converse is included in:

Theorem 10.2 Let $k$ be an integer, and let $A, B, L$ and $\eta$ be arbitrary complex constants such that (A.5) holds. The expression (8.2) representing the branch defined in $\{\zeta$ : $-\pi+k \pi<\arg \zeta<\pi+k \pi\}$ admits a finite number of zeros if and only if $A=-i B$ and $L-i \eta=n$, for some non-negative integer $n$. In particular, this implies that the modified Coulomb wave function $\psi_{+}(L, \eta ; \zeta)$ defined in $\{\zeta:-\pi+k \pi<\arg \zeta<\pi+k \pi\}$ admits a finite number of zeros if and only if $L-i \eta=n$ for some non-negative integer $n$. 
Proof The sufficiency follows from the above discussion immediately before Theorem 10.2 and if $A=-i B$ and $L-i \eta=n$, for some non-negative integer $n$, then (6.4) and hence (8.2) reduce to a $\psi_{+}$in terms of the generalized Bessel polynomial (A.13). The necessary part follows from $\S 6$ and the proof of Theorem 1.3 in $\S 8$ that if an entire function solution to equation (1.25) satisfies (1.3), then the (6.4) and hence (8.2) can only have a finite number of zeros. The Lemmas 6.1 and 6.4 imply that the coefficient $C=0$, say. This gives $A=-i B$, and that $L-i \eta=n$ (see (8.10)), for some non-negative integer $n$.

We remark that numerical studies that are related to the zeros of special cases of (8.2) can be found in $[16,20,39]$. We refer to $[17,20,27]$ and the references therein for the applications of the Coulomb wave functions.

\subsection{Bank and Laine's Theorem as a Hadamard-Type Result}

Bank and Laine obtained all the entire solutions of (1.1) with "few" zeros given by (1.4) and (1.5) using a purely complex analytic argument (Nevanlinna theory). In the special cases of (1.6) and (1.17) of (1.1), we have discovered that the polynomial components that appear in (1.8) and (1.18)-(1.19) in Theorems B and C are, in fact, respectively, the reverse and generalized Bessel polynomials. Our method is achieved by transforming the equations to two classes of confluent hypergeometic functions via the Lommel transformation, and by finding the conditions that characterize the finiteness of the zeros of (1.16) and (8.2) for arbitrary $A, B$ and the corresponding parameters.

We recall that the classical Hadamard theorem states that if a finite order entire function $f(z)$ assumes the value zero finitely often, then the factor $\Pi(z)$ in the Hadamard factorization $f(z)=\Pi(z) e^{g}$ is a polynomial. The new characterizations that we have found for (1.16) and (8.2) demonstrate that, if a combination of two linearly independent confluent hypergeometric functions (which are not entire in general)

$$
A \Psi(\zeta)+B \Phi(\zeta)
$$

assumes the value zero finitely often, then it reduces to a representation of the form $\Pi(z) e^{G}$ where $\Pi(z)$ is a polynomial. This resembles the Hadamard theorem.

\subsection{Remarks on Bessel Polynomials}

The Bessel polynomials appeared as early as 1873 in Hermite's work on transcendence of $e^{r / s}$ where $r / s$ is rational [23, IV], and have since reappeared in many different areas and applications [23]. The polynomials have been under intense study since the 1950 s and have a wealth of literature. We mention only that the Bessel polynomials appeared in a long and classical paper of Burchnall and Chaundy in 1931 [12] and independently in Krall and Frink [33] where the polynomials were formally named and systematically studied. 
The classical Sturm-Liouville type problems of differential equations study second order equations of the form:

$$
A(x) y^{\prime \prime}(x)+B(x) y^{\prime}(x)+\left(C(x)+\lambda_{n}\right) y(x)=0,
$$

where $A(x), B(x)$ and $C(x)$ are polynomials independent of the integer $n$ and only the coefficient $\lambda_{n}$ depends on $n$. Then Bochner [10] proved that there are only four types of equations of the above form which admit orthogonal polynomial solutions of exact degree $n$ (see also [38, pp. 107-108]), namely, the Jacobi, Laguerre, Hermite and Bessel polynomials.

The essential difference between the first three polynomials and the Bessel polynomials is that those three polynomials have real weight functions defined on or are on a subset of $\mathbf{R}$, while $y_{n}(x)$ (but not $\theta_{n}(x)$ ) have a complex weight function $e^{-2 / z}$ and that they are orthogonal with respect to the unit circle $[23,33]$.

\subsection{Remarks on Extensions to More General Equations}

It is clear from (3.8) and (7.3) that we could consider equations more general than (1.10) and (1.25), respectively. The solutions are given by (3.9) and (7.4), respectively. They are reduced to the solutions given in Theorems 1.2 and 1.6 when $\gamma=0$. However, since no essential new information concerning the value distribution of the solutions can be obtained by the previous method, we choose not to pursue further the cases where $\gamma \neq 0$ for these equations.

We also have not given any results for the equation (1.1) when $\ell \geq 3$ in (1.2) as in Theorems 1.2, 1.3 and 1.6. This is because of the corresponding equations obtained after the Lommel transformation are given by the generalized Bessel equations in the form (3.2) where $n^{\prime}=0$ and $n \geq 3$. The equation has, as in the case of confluent hypergeometric equations, a regular singularity at $z=0$ and an irregular singularity at $z=\infty$. However, as we have mentioned earlier, there does not seem to have been a suitable theory and tools for the generalized Bessel equations as in the case of Bessel functions.

Bank [3] and Gao [22] proved that if either $\ell$ or $k$ in (1.2) is positive and odd, then any solution $f$ of (1.1) will violate (1.3). The corresponding equation (3.2) has an irregular singularity at $z=0$ and so it is no longer confluent hypergeometric. This is reflected in Bank and Gao's results $[3,22]$ that no solution $f(z)$ of the equation (3.2) can have $\lambda(f)<+\infty$. That is, no (orthogonal) polynomial component as in (1.4) and (1.5) can be found. The remaining case of (1.2) when both $\ell$ and $k$ are positive and even implies that the transformed equation (3.2) has irregular singular points at 0 and $\infty$.

All the above cases pose a major barrier to extending our method and argument to (1.2) outside the two classes (1.10) and (1.25) studied here.

\subsection{Remarks on Physical Applications of the Hankel and Coulomb Wave Functions}

The problem of a certain scattering amplitude in electromagnetic theory having poles at the values of $\nu$ for which $H_{\nu}^{(1)}(z)=0$ was discovered by G. N. Watson [41] (see 
also [32]). The idea was later used by T. Regge in the study of quantum-mechanical potential scattering $[15,19,27,32,37]$.

\section{A Appendix}

Following [2, p. 209], we have for $-\pi<\arg x<2 \pi$,

$$
H_{\nu}^{(1)}(x)=\left(\frac{2}{\pi x}\right)^{\frac{1}{2}} e^{i\left(x-\frac{1}{2} \nu \pi-\frac{1}{4} \pi\right)}\left[\sum_{m=0}^{p-1} \frac{\left(\frac{1}{2}-\nu\right)_{m}\left(\frac{1}{2}+\nu\right)_{m}}{(2 i x)^{m} m !}+R_{p}^{(1)}(x)\right]
$$

and for $-2 \pi<\arg x<\pi$,

$$
H_{\nu}^{(2)}(x)=\left(\frac{2}{\pi x}\right)^{\frac{1}{2}} e^{-i\left(x-\frac{1}{2} \nu \pi-\frac{1}{4} \pi\right)}\left[\sum_{m=0}^{p-1} \frac{\left(\frac{1}{2}-\nu\right)_{m}\left(\frac{1}{2}+\nu\right)_{m}}{(2 i x)^{m} m !}+R_{p}^{(2)}(x)\right]
$$

where

$$
R_{p}^{(1)}(x)=O\left(x^{-p}\right) \quad \text { and } \quad R_{p}^{(2)}(x)=O\left(x^{-p}\right)
$$

as $x \rightarrow+\infty$, uniformly in $-\pi+\delta<\arg x<2 \pi-\delta$ and $-2 \pi+\delta<\arg x<\pi-\delta$, respectively. The two expansions are valid in $-\pi<\arg x<\pi$ simultaneously. We also note that the expansions are divergent [40, p. 194].

Since the Coulomb wave functions $F_{L}(\eta, x)$ and $G_{L}(\eta, x)$ are defined in terms of the Kummer functions e.g., (6.1), so they are also confluent hypergeometric functions. We record here the definitions of the Coulomb phase shift function

$$
\left.\sigma_{L}(\eta)=\frac{1}{2 i}[\log \Gamma(L+1+i \eta))-\log \Gamma(L+1-i \eta)\right]
$$

It is a well-defined function under the restriction [17, (2.3a), (2.3b)]

$$
\begin{gathered}
L+1 \pm i \eta \neq 0, \quad-\pi<\arg (L+1 \pm i \eta)<\pi, \\
\log \Gamma(L+1 \pm i \eta) \text { is real when } L \text { is real and } \leq-1 .
\end{gathered}
$$

We refer to [17, p. 6164] for the details about the phase shift function. In addition we also define the phase by

$$
x(L, \eta)=\left(L+\frac{1}{2}\right) \pi+\sigma_{-L-1}(\eta)-\sigma_{L}(\eta) .
$$

We now state the asymptotic expansions for $\psi_{ \pm}(x)$ which are taken from [17, (8.5a-b), p. 6163],

$$
\psi_{+}(L, \eta ; x)=\frac{e^{i \chi(L, \eta ; x)}}{\Gamma(L+1+i \eta)}\left[\sum_{k=0}^{p-1}\left(\begin{array}{c}
L-i \eta \\
k
\end{array}\right)\left(\frac{-1}{2 i x}\right)^{k} \Gamma(L+1+i \eta+k)+L_{p}^{(+)}(x)\right]
$$


for $-\pi<\arg x<2 \pi$, and

$$
\psi_{-}(L, \eta ; x)=\frac{e^{-i \chi(L, \eta ; x)}}{\Gamma(L+1-i \eta)}\left[\sum_{k=0}^{p-1}\left(\begin{array}{c}
L+i \eta \\
k
\end{array}\right)\left(\frac{1}{2 i x}\right)^{k} \Gamma(L+1-i \eta+k)+L_{p}^{(-)}(x)\right] \text {, }
$$

for $-2 \pi<\arg x<\pi$ where

$$
L_{p}^{(+)}(x)=O\left(x^{-p}\right) \quad \text { and } \quad L_{p}^{(-)}(x)=O\left(x^{-p}\right) .
$$

Thus the above asymptotic expansions are valid uniformly in $-\pi<\arg x<\pi$. Using the following analytic continuation formula

(A.10) $\psi_{+}(L, \eta ; \zeta)=e^{2 \pi \eta} \psi_{+}\left(L, \eta ; \zeta e^{-2 \pi i}\right)-e^{2 \pi \eta}\left(1-e^{-2 \pi \eta(L-i \eta)}\right) \psi_{-}\left(L, \eta ; \zeta e^{-2 \pi i}\right)$

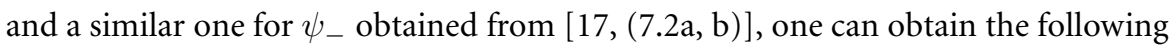
more general asymptotic formula [17, §VIII] for $(2 m-2) \pi+\varepsilon<\arg z<2 m \pi-\varepsilon$ $(m \in \mathbb{Z})$ given in $[17,(8.8 \mathrm{a})]$ :

$$
\begin{aligned}
\psi_{+}(L, \eta ; \rho) \sim \frac{e^{-2(m-1) \pi \eta}}{\sin (2 \pi L)} & \left\{\sin (2 m \pi L)-\sin [2(m-1) \pi L] e^{-2 \pi \eta}\right\} \cdot e^{i \chi(L, \eta ; \rho)} \\
& -\frac{e^{+2(m+1) \pi \eta}}{\sin (2 \pi L)} \sin (2 m \pi L)\left\{1-e^{-2 \pi i(L-i \eta)}\right\} \cdot e^{-i \chi(L, \eta ; \rho)} .
\end{aligned}
$$

A corresponding asymptotic expansion for $\psi_{-}$in $(2 m-2) \pi+\varepsilon<\arg z<2 m \pi-\varepsilon$ $(m \in \mathbb{Z})$ can be found in $\left[17,\left(8.8 \mathrm{a}^{\prime}\right)\right]$.

We also need the connections between the Coulomb wave functions and the Whittaker functions $[17$, p. 6146]:

$$
\psi_{+}(L, \eta, x)=\exp \left\{i\left[\sigma_{L}(\eta)-(L+i \eta) \pi / 2\right]\right\} W_{-i \eta, L+1 / 2}\left(2 x e^{-i \frac{\pi}{2}}\right) .
$$

In particular, we further note that the formulae [23, p. 38, (vii), (viii)] reveal that the Whittaker function is closely related to the Bessel and the generalized Bessel polynomials. We have

$$
y_{n}(x ; a, b)=e^{\frac{b}{2 x}}\left(\frac{x}{b}\right)^{1-a / 2} W_{1-a / 2,(a-1) / 2+n}\left(\frac{b}{x}\right)
$$

and

$$
y_{n}(x)=y_{n}(x ; a, b)=e^{\frac{1}{x}} W_{0, n+1 / 2}\left(\frac{2}{x}\right)
$$

when $a=b=2$. We note here that the Whittaker function is again a confluent hypergeometric function. We refer to $[11,42]$ for the details about the functions.

Acknowledgement The authors would like to acknowledge useful communications with J. Bustoz, S. Skorokhodov, J. Sesma concerning the zeros of Hankel functions and S. Yngve for information concerning the Coulomb wave functions. In the original version of the manuscript, the authors used a simpler way to prove Lemma 2.1. We thank Walter Hayman for his suggestion of the proof in the present form. Finally, we thank the referee for useful comments and for making the formulation of Theorem 9.1 more accurate as in the present form. 


\section{References}

[1] M. Abramowitz and I. A. Stegun (eds.), Handbook of Mathematical Functions. National Bureau of Standards, Applied Mathematics Series 55, Washington, 1964.

[2] G. E. Andrews, R. Askey, and R. Roy, Special Functions. Encyclopedia of Mathematics and its Applications 71, Cambridge University Press, Cambridge, 1999.

[3] S. B. Bank, Three results in the value-distribution theory of solutions of linear differential equations. Kodai Math. J. 9(1986), no. 2, 225-240.

[4] On the explicit determination of certain solutions of periodic differential equations. Complex Variables Theory Appl. 23(1993), no. 1-2, 101-121.

[5] A note on the oscillation of solutions of periodic linear differential equations. Czechoslovak Math. J. 44(119) (1994), no. 1, 91-107.

[6] S. B. Bank and I. Laine, On the oscillation theory of $f^{\prime \prime}+A f=0$ where $A$ is entire. Trans. Amer. Math. Soc. 273(1982), no. 1, 351-363.

[7] Representations of solutions of periodic second order linear differential equations. J. Reine Angew. Math. 344(1983), 1-21.

[8] S. B. Bank, I. Laine, and J. K. Langley, On the frequency of zeros of solutions of second order linear differential equations. Results Math. 10(1986), 8-24.

[9] Oscillation results for solutions of linear differential equations in the complex domain. Results Math. 16(1989), no. 1-2, 3-15.

[10] S. Bochner, Über Sturm-Liouvillsche polynomsysteme. Math. Z. 29(1929), no. 1, 730-736.

[11] H. Buchholz, The Confluent Hypergeometric Function With Special Emphasis on Its Applications. Berlin, Springer-Verlag, 1969.

[12] J. L. Burchnall and T. W. Chaundy, The commutative ordinary differential operators II - The identity $P^{n}=Q^{m}$. Proc. Royal Soc. London Ser. A 134 (1931), 471-485.

[13] Y. M. Chiang, On the complex oscillation of $y^{\prime \prime}+\left(e^{z}-K\right) y=0$ and a result of Bank, Laine and Langley. In: Computational Methods and Function Theory, Ser. Approx. Decompos. 5, World Scientific, River Edge, NJ, 1995, 125-134.

[14] On the zero-free solutions of linear periodic differential equations in the complex plane. Results Math. 38(2000), no. 3-4, 213-225.

[15] A. Cruz, J. Esparza, and J. Sesma, Zeros of the Hankel functions of real order out of the principal Riemann sheet. J. Comp. Appl. Math. 37(1991), no. 1-3, 89-99.

[16] A. R. Curtis, Coulomb Wave Functions. Royal Society Mathematical Tables II, Cambridge University Press, New York, 1964.

[17] A. Dzieciol, S. Yngve, and P. O. Fröman, Coulomb wave functions with complex values of the variable and the parameters. J. Math. Physics, 40(1999), no. 12, 6145-6166.

[18] A. Erdélyi, ed, Higher Transcendental Functions. Vol. II, McGraw-Hill, New York 1953.

[19] J. Esparza and J. Sesma, Regge Trajectories for the inverse square potential. J. Math. Physics, 11(1970), 3245-3250

[20] J. Esparza, J. L. López, and J. Sesma, Zeros of the Whittaker function associated to Coulomb waves. IMA J. Appl. Math. 63(1999), no. 1, 71-87.

[21] M. Frei, Über die subnormalen Lösungen der Differentialgleichung $w^{\prime \prime}+e^{-z} w^{\prime}+$ konst. $w=0$. Comment Math. Helv. 36(1961), 1-8.

[22] S. Gao, Some results on the complex oscillation theory of periodic second-order linear differential equations. Kexue Tongbao 33(1988), no. 13, 1064-1068.

[23] E. Grosswald, Bessel Polynomials. Lecture Notes in Math. 698, Springer-Verlag, Berlin, 1978.

[24] G. G. Gundersen and E. M. Steinbart, Subnormal solutions of second order linear differential equations with periodic coefficients. Results Math. 25(1994), no. 3-4, 270-289.

[25] W. K. Hayman, Meromorphic Functions. Clarendon Press, Oxford, 1964.

[26] E. Heine, Handbuch der Kugelfunctionen, Theorie und Anwendungen. 1. Second ed. Reimer, Berlin, 1878.

[27] M. H. Hull, Jr. and G. Breit, Coulomb Wave Functions. Handbuch der Physik 41/1, Springer, Berlin, 1959, pp. 408-465.

[28] E. Hille, Ordinary Differential Equations in the Complex Domains. Wiley-Interscience, 1976.

[29] M. E. H. Ismail, An electrostatic model for zeros of general orthogonal polynomials. Pacific J. Math. 193(2000), no. 2, 355-369.

[30] M. E. H. Ismail, S. S. Lin and S. S. Roan, Bethe Ansatz equation of XXZ model and q-Sturm-Louville problems (to appear).

[31] E. Kamke, Differentialgleichungen, Lösungsmethoden und Lösungen. Akademische Verlagsgesellschaft, Leipzig, 1943. 
[32] J. B. Keller, S. I. Rubinow and M. Goldstein, Zeros of Hankel functions and poles of scattering amplitudes. J. Math. Physics 4(1963), 829-832.

[33] H. L. Krall and O. Frink, A new class of orthogonal polynomials: The Bessel polynomials. Trans. Amer. Math. Soc. 65(1949), 100-115.

[34] I. Laine, Nevanlinna Theory and Complex Differential Equations. de Gruyter Studies in Mathematics 15, Walter de Gruyter, Berlin, 1993.

[35] E. Lommel, Zur Theorie der Bessel'schen Functionen. Math. Ann. 3(1871), no. 4, 475-487.

[36] K. Pearson, On the solution of some differential equations by Bessel's functions. Messenger of Math. IX(1880), 127-131.

[37] S. L. Skorokhodov, On the computation of complex zeros of the modified Bessel function of the second kind. Soviet Math. Dokl. 31, No. 5, (1985), 78-81.

[38] G. Szegö, Orthogonal Polynomials. Fourth ed. American Mathematical Society Colloquium Publications 23, American Mathematical Society, Providence, RI, 1975,

[39] I. J. Thompson and A. R. Barnett, Coulomb and Bessel Functions of complex arguments and order. J. Comput. Phys. 64(1986), no. 2, 490-509.

[40] G. N. Watson, A Treatise on the Theory of Bessel Functions. Second edition. Cambridge University Press, Cambridge, 1944.

[41] The defraction of electric waves by the earth. Proc. Roy. Soc. London A95(1918), No. 666 83-99.

[42] E. T. Whittaker and G. N. Watson, A Course of Modern Analysis. Fourth ed. Cambridge University Press, Cambridge, 1927, (reprinted in 1996).

[43] H. Wittich, Subnormale Lösungen der Differentialgleichung $w^{\prime \prime}+p\left(e^{z}\right) w^{\prime}+q\left(e^{z}\right) w=0$. Nagoya Math. J. 30(1967), 29-37.

Department of Mathematics

Hong Kong University of Science and Technology

Clear Water Bay, Hong Kong

P.R. China

e-mail:machiang@ust.hk
Department of Mathematics University of Central Florida

Orlando, FL 32816

U.S.A.

e-mail: ismail@math.ucf.edu 